\title{
COMPORTAMIENTO A CORTANTE DE MUROS DE CONCRETO PARA VIVIENDA
}

\author{
Julián Carrillo $^{(1)}$ y Sergio M. Alcocer ${ }^{(2)}$
}

\begin{abstract}
RESUMEN
La ausencia de reglamentos adecuados para diseñar vivienda de baja altura ha promovido que el desempeño sísmico de estas estructuras haya sido deficiente durante los últimos sismos ocurridos en países latinoamericanos. Actualmente, la construcción de viviendas de concreto es una de las opciones más eficientes. Debido al potencial de rigidez y resistencia lateral de estructuras con muros concreto, las demandas de fuerzas y de desplazamientos son limitadas y, por tanto, se emplean muros con resistencia baja de concreto, espesor reducido, cuantías de refuerzo menores que la mínima especificada en el reglamento y mallas de alambre soldado como refuerzo a cortante en el alma. Considerando las características particulares de los muros, los modelos analíticos y los requisitos de los reglamentos disponibles no son directamente aplicables. Con base en los resultados de un extenso programa de investigación experimental y analítico, se desarrollan ecuaciones para estimar la resistencia máxima al cortante. Adicionalmente, utilizando un enfoque de diseño por desempeño, se proponen valores representativos de los parámetros principales para diseño sísmico. Asimismo, se presentan requisitos de refuerzo a cortante en función de la demanda sísmica sobre los muros.
\end{abstract}

Palabras Clave: muros de concreto, resistencia a cortante, vivienda de baja altura, concreto ligero, diseño sísmico por desempeño.

\begin{abstract}
The lack of suitable building codes for designing low-rise housing has promoted that the performance of such structures has been inadequate during the most recent earthquakes occurred in Latin American countries. Construction of concrete housing is currently one of the most accomplished techniques. Due to the potential lateral stiffness and strength of concrete wall structures, forces and displacements demands are limited, thus leading to the use of walls with low concrete strength, small thickness, web steel ratio smaller than the minimum amount prescribed by the code and shear web reinforcement made of welded wire meshes. Considering the particular wall characteristics, existing analytical models and requirements in current codes are not directly applicable. Based on an extensive experimental and analytical research program, equations for estimating the maximum shear strength are developed. In addition, using a performance-based seismic design approach, representative values of the main parameters for seismic design are proposed. Shear reinforcement requirements depending on the seismic demand are also recommended.
\end{abstract}

Artículo ganador de séptimo concurso nacional de tesis de doctorado, recibido el 12 de noviembre de 2011 y aprobado para su publicación el 2 de diciembre de 2011

(1) Universidad Militar Nueva Granada, UMNG, Departamento de Ingeniería Civil, Bogotá, Colombia; wjcarrillo@gmail.com

(2) Instituto de Ingeniería, Universidad Nacional Autónoma de México, UNAM, México, D.F.; salcocerm@ii.unam.mx 
Key Words: concrete walls, shear strength, low-rise housing, lightweight concrete, performance-based seismic design.

\section{INTRODUCCIÓN}

Los últimos sismos ocurridos en Colombia, Haití, México y Perú (Pujol et al., 1999; Eberhard et al., 2010; Johansson et al., 2007), de nuevo han evidenciado la ausencia de recomendaciones y reglamentos de diseño eficientes para la construcción de viviendas de baja altura. Infortunadamente, la población con recursos económicos limitados resulta ser la más afectada tras la ocurrencia de desastres naturales (Carrillo, 2010b). Una de las opciones más eficientes para la construcción de viviendas de interés social es el desarrollo de conjuntos habitacionales con viviendas de concreto en su totalidad (cimentación, muros, losas de entrepiso y techos). Si bien el costo unitario de los materiales empleados en los muros de concreto es superior al de la mampostería tradicional, el ahorro económico y la edificación sustentable se logran con la rapidez de construcción y el uso de concretos de características especiales. Por ejemplo, la utilización de sistemas de cimbras de acero, así como mallas de alambre soldado como refuerzo a cortante en el alma, garantizan la disminución del tiempo y el costo de la vivienda. Adicionalmente, el empleo de concretos de tipo ligero y autocompactable promueve el ahorro de energía y prolonga la vida útil de las viviendas. Para este tipo de viviendas, usualmente se utilizan muros con resistencia baja de concreto, espesor y cuantías de refuerzo reducidas y, en la mayoría de los casos, mallas de alambre soldado como refuerzo a cortante en el alma. Tomando en cuenta las características particulares de los muros de concreto utilizados en las viviendas de baja altura, las recomendaciones de diseño vigentes en los reglamentos no son directamente aplicables. Además, los parámetros de diseño sísmico que se proponen en los reglamentos vigentes están enfocados principalmente al diseño de muros de concreto para edificios de mediana o gran altura (Duffey et al., 1994a, 1994b).

Por tanto, se llevó a cabo un extenso programa de investigación con la finalidad de proponer criterios de análisis y diseño sísmico aplicables a muros de concreto para viviendas de baja altura. El programa experimental incluyó 39 ensayos cuasi-estáticos y dinámicos de muros con diferente relación de aspecto y sistemas de muros con aberturas. Las variables estudiadas fueron el tipo de concreto (peso normal, peso ligero y autocompactable), la cuantía de acero de refuerzo a cortante en el alma (0\%, 0.125\% y $0.25 \%$ ) y el tipo de refuerzo (barras corrugadas y malla de alambre soldado). A partir de estudios analíticos y de resultados experimentales, se proponen ecuaciones para estimar la resistencia máxima al cortante y valores representativos de los parámetros principales para diseño sísmico (factor de comportamiento sísmico y distorsiones permisibles de entrepiso). Asimismo, se recomiendan valores de las cuantías mínimas de refuerzo a cortante en función de la demanda sísmica sobre los elementos, así como requisitos por cambios volumétricos y el refuerzo por integridad estructural. Considerando que en los últimos años el diseño sísmico por resistencia ha experimentando una reevaluación trascendental, con el énfasis cambiando de "resistencia” a "desempeño", los valores de distorsión permisible se presentan en el formato de la metodología de diseño sísmico por desempeño.

\section{PROGRAMA EXPERIMENTAL}

El programa experimental del proyecto incluyó el ensayo de 39 muros aislados de concreto: 33 muros ensayados bajo carga lateral cuasi-estática monótona y cíclica-reversible (Flores et al., 2007; Sánchez, 2010) y 6 muros a escala levemente reducida ensayados dinámicamente en mesa vibradora (Carrillo, 2010a). Las variables de estudio se obtuvieron de las empleadas con más frecuencia en la práctica del diseño y construcción de viviendas de concreto en México (tabla 1). 


\section{Geometría y refuerzo}

En la figura 1 se muestran la geometría nominal y la configuración característica del refuerzo de los muros con relación de aspecto $\left(h_{w} / l_{w}\right)$ igual a uno, ensayados bajo carga lateral cuasi-estática. La configuración del refuerzo de todos los especímenes se puede encontrar en Flores et al. (2007), Sánchez (2010) y Carrillo (2010). Con el propósito de que las solicitaciones encontradas durante los ensayos dinámicos no superaran las características de la mesa vibradora, y que los modelos fueran representativos del prototipo de vivienda, los especímenes ensayados dinámicamente se diseñaron en escala 1:1.25 (80\% del tamaño real). Tomando en cuenta que la escala de los modelos ensayados en mesa vibradora fue sólo levemente reducida (1:1.25), se seleccionó un modelo de similitud simple (Tomazevic y Velechovsky, 1992). En este tipo de similitud, los modelos se construyen con los mismos materiales que el prototipo, es decir, no se alteran las propiedades de los materiales, sólo las dimensiones de los modelos. La geometría de estos especímenes se estableció a partir de la geometría de los muros ensayados bajo carga lateral cíclica cuasi-estática.

Tabla 1 Descripción de las variables de estudio

\begin{tabular}{ll}
\hline \multicolumn{1}{c}{ Variable } & \multicolumn{1}{c}{ Descripción } \\
\hline Relación de aspecto: & $\begin{array}{l}\text { cuadrados }\left(h_{w} / l_{w}=1\right) \text {, robustos }\left(h_{w} / l_{w}=0.5\right) \text {, esbeltos }\left(h_{w} / l_{w}=2\right) \text { y muros con aberturas } \\
\text { (en escala natural) fue igual a } 100 \mathrm{~mm} \text { y } 2.4 \mathrm{~m} \text {, respectivamente. Por tanto, para } \\
\text { alcanzar una determinada relación de aspecto, se varió la longitud del muro }\left(l_{w}\right)\end{array}$ \\
\hline Tipo de concreto: & $\begin{array}{l}\text { peso normal, peso ligero y autocompactable, con resistencia nominal a la } \\
\text { compresión, } f_{c}{ }^{\prime}=15 \mathrm{MPa} \text {. }\end{array}$ \\
\hline $\begin{array}{l}\text { Cuantía de refuerzo a } \\
\text { cortante en el alma } \\
\text { (vertical y horizontal): }\end{array}$ & $\begin{array}{l}100 \% \rho_{\min }(0.25 \%), 50 \% \rho_{\min }(0.125 \%), 0=\text { sin refuerzo en el alma. El porcentaje de } \\
\text { la cuantía se expresó como fracción de la cuantía mínima }\left(\rho_{\min }\right) \text { estipulada en las } \\
\text { NTC-C }(2004) .\end{array}$ \\
\hline $\begin{array}{l}\text { Tipo de refuerzo a } \\
\text { cortante en el alma: }\end{array}$ & $\begin{array}{l}\text { barras corrugadas de acero con esfuerzo de fluencia nominal, } f_{y}=412 \text { MPa y malla } \\
\text { de alambre soldado con esfuerzo de fluencia nominal, } f_{y}=491 \text { MPa. }\end{array}$ \\
\hline Tipo de ensayo: & $\begin{array}{l}\text { cuasi-estático monótono, cuasi-estático cíclico-reversible y dinámico en mesa } \\
\text { vibradora. }\end{array}$ \\
\hline
\end{tabular}

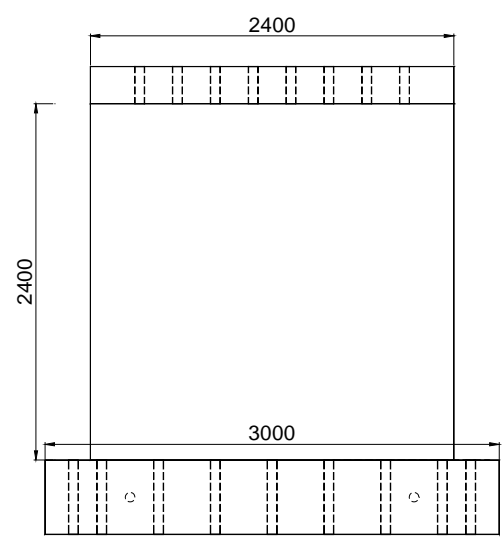

(a) Geometría (dimensiones en mm)
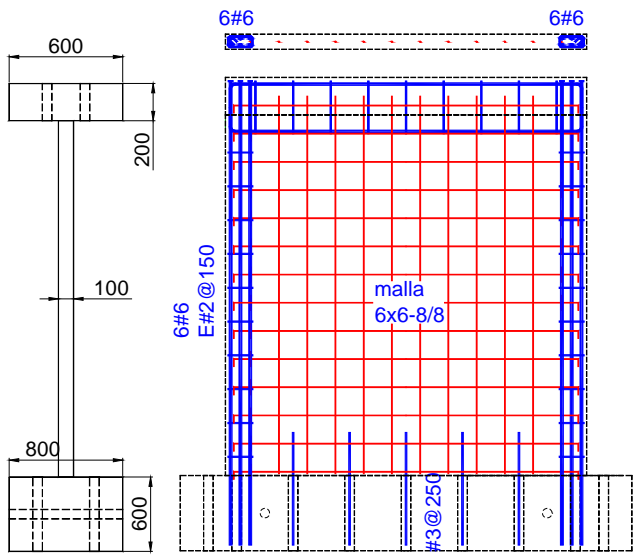

(b) Con malla de alambre soldado

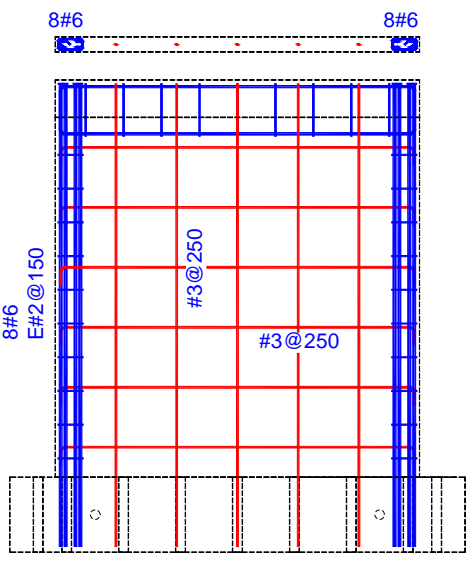

(c) Con barras corrugadas

Figura 1 Geometría y refuerzo de muros con $h_{w} / l_{w}=1.0$

Los especímenes se desplantaron sobre una viga de cimentación rígida, con el propósito de evitar la fisuración de los especímenes durante el transporte. Este elemento también fue utilizado para sujetar los 
especímenes a la losa de reacción (ensayos cuasi-estáticos) o a la plataforma de la mesa vibradora (ensayos dinámicos), así como para el anclaje de las barras verticales. En la parte superior, los modelos contaron con una losa que funcionó como el elemento para conectar el dispositivo de los ensayos dinámicos o los gatos hidráulicos de doble acción (ensayos cuasi-estáticos), así como para el anclaje de las barras verticales. El acero de refuerzo del alma de los muros se colocó en una sola capa en la mitad del espesor y se utilizaron las mismas cuantías en las direcciones horizontal y vertical. Teniendo en cuenta la geometría y el sistema estructural de las viviendas de concreto de baja altura, la rotación superior de los muros está restringida y, por tanto, el comportamiento de los muros está gobernado por deformaciones de cortante. De esta manera, el refuerzo longitudinal en los elementos de borde se dispuso para evitar falla por flexión diferente a la falla por cortante predominante en este tipo de elementos.

\section{Propiedades mecánicas de los materiales}

En las tablas 2 y 3 se presentan los valores promedio de las principales propiedades mecánicas del concreto y del acero de refuerzo en el alma del muro, respectivamente. Para el concreto, estas propiedades fueron obtenidas en una fecha cercana al ensayo de los modelos. La resistencia nominal a la compresión del concreto fue igual a $15 \mathrm{MPa}$ y la resistencia nominal de fluencia de las barras corrugadas y los alambres de malla fueron 412 y $491 \mathrm{MPa}$, respectivamente.

Tabla 2 Propiedades mecánicas del concreto

\begin{tabular}{llccccc}
\hline \multicolumn{1}{c}{ Propiedad } & & Peso normal & & Peso ligero & & Autocompactable \\
\cline { 1 - 1 } \cline { 5 - 6 } \cline { 5 - 6 } Resistencia a compresión, $f_{c}, \mathrm{MPa}$ & & $16.0-24.7$ & & $10.8-26.0$ & & $22.0-27.1$ \\
Módulo de elasticidad, $E_{c}, \mathrm{MPa}$ & & $8430-14750$ & & $6700-10790$ & & $8900-11780$ \\
Resistencia a tensión diametral, $f_{t}, \mathrm{MPa}$ & & $1.55-2.20$ & & $1.14-1.76$ & & $1.58-1.98$ \\
Resistencia a flexión, $f_{r}, \mathrm{MPa}$ & & $2.32-3.75$ & & $1.43-3.29$ & & $2.27-2.48$ \\
Peso específico (seco), $\gamma, \mathrm{kN} / \mathrm{m}^{3}$ & & $18.8-20.3$ & & $15.2-18.3$ & & 18.9 \\
\hline
\end{tabular}

En la tabla 3 se observa que el alargamiento de las mallas utilizadas en la construcción de los especímenes fue menor que el estipulado en la norma NMX-B-253 (2006). El comportamiento de los alambres de la malla estuvo caracterizado por la fractura del material con un leve incremento de deformación [figura 3(a)]. Teniendo en cuenta esta preocupación, Rico et al. (2011) desarrollaron un programa de investigación experimental para estudiar las propiedades mecánicas de los alambres y la soldadura utilizada en la fabricación de la malla de alambre soldado disponible en la zona metropolitana de la ciudad de México. En dicho estudio se confirmó que las mallas estudiadas no cumplen con el alargamiento mínimo especificado en la norma correspondiente vigente.

Tabla 3 Propiedades mecánicas del acero de refuerzo

\begin{tabular}{|c|c|c|c|}
\hline Ubicación en el muro & $\begin{array}{c}\text { Extremos: } \\
\text { Barra corrugada }\end{array}$ & $\begin{array}{c}\text { Alma: } \\
\text { Barra corrugada }\end{array}$ & $\begin{array}{c}\text { Alma: } \\
\text { Alambre de malla }\end{array}$ \\
\hline Resistencia de fluencia, $f_{y}, \mathrm{MPa}$ & $411-456$ & $435-447$ & $605-630$ \\
\hline Resistencia última, $f_{\text {su }}, \mathrm{MPa}$ & $656-721$ & $659-672$ & $687-700$ \\
\hline Alargamiento, \% & $9.1-16.0$ & $10.1-11.0$ & $1.4-1.9$ \\
\hline
\end{tabular}

\section{Tipos y configuración de ensayos}

En los ensayos dinámicos, la solicitación horizontal estuvo representada por registros sísmicos aplicados a una mesa vibradora sobre la cual se sujetaron los especímenes. Con el fin de estudiar el comportamiento de los muros para diferentes estados límite, desde el inicio del agrietamiento hasta el colapso, los modelos ensayados dinámicamente fueron sometidos a tres niveles de amenaza sísmica 
utilizando acelerogramas naturales y sintéticos. Los registros fueron representativos de la zona de subducción del pacífico mexicano. En los ensayos dinámicos se adicionó la masa necesaria para que el periodo de vibración inicial de los modelos fuera similar al periodo de las viviendas típicas de baja altura. Para establecer el periodo de vibración se desarrollaron modelos analíticos que fueron calibrados con resultados de pruebas de vibración ambiental (Carrillo y Alcocer, 2011a). La masa se ubicó sobre un dispositivo externo (Carrillo y Alcocer, 2011b) y la carga vertical se aplicó utilizando lingotes de plomo sujetos a la viga de carga situada en el extremo superior de los modelos.

En los ensayos cuasi-estáticos, como de forma convencional, la carga horizontal se aplicó a nivel de la losa mediante gatos hidráulicos de doble acción. Los ciclos de carga se aplicaron con una repetición para cada incremento. Los primeros ciclos se controlaron por carga hasta el agrietamiento. Los dos primeros ciclos se aplicaron hasta el $25 \%$ de la carga de agrietamiento calculada. El siguiente incremento se dio hasta el 50\% de la misma, con su respectiva repetición. A continuación se aplicó un incremento hasta llegar a la carga de agrietamiento real. Posteriormente, la historia de controló por distorsión con aumentos de 0.002 en cada incremento y una repetición para cada distorsión. Carrillo (2010) presenta los detalles del protocolo de los ensayos cuasi-estáticos y de los registros sísmicos utilizados en los ensayos dinámicos.

\section{Instrumentación de los especímenes}

Para medir la respuesta de los especímenes, los muros fueron instrumentados interna y externamente. La instrumentación interna se diseñó para adquirir información de la respuesta local del refuerzo utilizando deformímetros adheridos al acero de refuerzo, específicamente, para evaluar la contribución del acero de refuerzo. La instrumentación externa fue planeada para conocer la respuesta global por medio de transductores de desplazamiento, aceleración y carga. Adicionalmente, se utilizó un sistema de medición óptico de desplazamientos, el cual utiliza diodos emisores de luz (LED, por sus siglas en inglés). En la figura 2 se muestran la instrumentación característica de los muros con relación de aspecto $\left(h_{w} / l_{w}\right)$ igual a uno, ensayados en mesa vibradora.

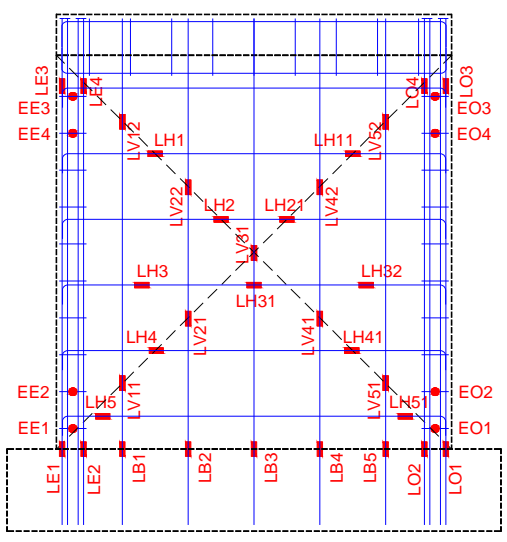

(a) Interna (barras corrugadas)

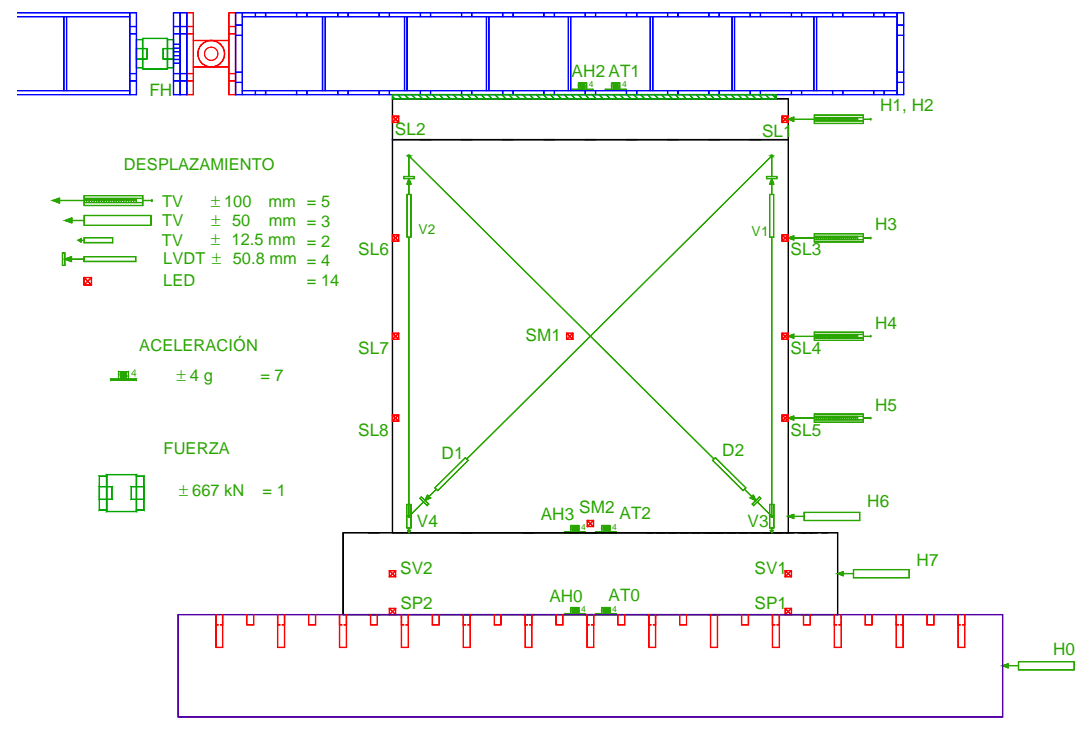

(b) Externa

Figura 2 Instrumentación de muros con $h_{w} / l_{w}$, ensayados en mesa vibradora 


\section{CAPACIDAD DE RESISTENCIA A CORTANTE}

Actualmente existen varios modelos analíticos para predecir la resistencia a cortante de muros de concreto. Tomando en cuenta las características particulares de los muros de concreto para viviendas de baja altura, la mayoría de las recomendaciones y ecuaciones de predicción de los modelos disponibles no es directamente aplicable para diseñar viviendas con las características estudiadas. En general, las principales limitaciones de dichos modelos son: (a) se calibraron para un intervalo muy amplio de los parámetros que controlan el comportamiento de muros de concreto; no obstante, para las viviendas analizadas, la mayoría de los parámetros varía en un intervalo limitado (resistencias bajas del concreto, espesor reducido de muros, esfuerzo axial bajo, cuantías de refuerzo reducidas, entre otras); (b) no se incluye el comportamiento de muros reforzados con malla de alambre soldado, en los cuales, el alargamiento de los alambres y la capacidad de desplazamiento del muro es un parámetro fundamental para diseño sísmico por desempeño; (c) todas las metodologías se han calibrado utilizando resultados de especímenes ensayados bajo carga lateral cuasi-estática, es decir, no se incluyen los efectos de la velocidad de aplicación de carga, el número de ciclos, los parámetros acumulados de ductilidad y energía disipada (Carrillo y Alcocer, 2010; Carrillo et al., 2009), así como los efectos dinámicos de interacción entre la carga axial (compresión y/o tensión), el momento flexionante y la fuerza cortante en el plano; y (d) algunos han sido planteados para fines académicos, es decir, su formato no es realmente práctico y sencillo para ser utilizadas con fines de diseño.

A partir de lo anterior, se desarrolló un modelo de predicción de resistencia para muros de concreto en viviendas de baja altura. De modo similar al enfoque de los reglamentos de diseño vigentes, el modelo aquí propuesto pretende que las fallas por tensión diagonal ocurran antes que las fallas por compresión diagonal o por deslizamiento y, por tanto, la resistencia a cortante está relacionada con el mecanismo de falla por tensión diagonal. Sin embargo, se deben revisar las resistencias a cortante asociadas a los mecanismos de falla de compresión diagonal y por deslizamiento.

\section{Tensión y compresión diagonal}

A partir del análisis de la información experimental de los muros aquí estudiados, junto con las tendencias y observaciones de los modelos disponibles, se propone calcular la resistencia a cortante de muros de concreto para vivienda de baja altura por medio de la ecuación 1. El primer término de la ecuación representa la resistencia por tensión diagonal, que es igual a la suma de la contribución del concreto $\left(V_{c}\right)$ más la contribución a la resistencia del refuerzo horizontal del alma del muro $\left(V_{s}\right)$. El segundo término simboliza el límite superior de la resistencia a cortante para prevenir un modo de falla por compresión diagonal.

$$
V_{\max }=\left[\alpha_{1} \sqrt{f_{c}^{\prime}}+\eta_{h} \rho_{h} f_{y h}\right] A_{w} \leq \alpha_{2} \sqrt{f_{c}^{\prime}} A_{w}
$$

donde $f_{c}$ ' es la resistencia nominal a la compresión del concreto, $\rho_{h}$ es la cuantía de refuerzo horizontal en el alma de muro, $\eta_{h}$ es el factor de eficiencia de $\rho_{h}, A_{w}$ es el área total de la sección transversal del muro $\left(A_{w}=t_{w} \times l_{w}\right)$ y, $\alpha_{1}$ y $\alpha_{2}$ son factores para determinar la contribución del concreto a la resistencia por tensión y compresión diagonal, respectivamente. Para facilitar los cálculos, la ecuación 1 se calibró con el área total del muro, $A_{w}$. En la ecuación 1 no se incluyó el factor de modificación de las propiedades mecánicas del concreto de peso ligero que se especifica de forma implícita en el reglamento NTC-C (2004) y de forma explícita en ACI-318 (2008), pues las tendencias de los resultados experimentales demostraron que este factor no se debe aplicar al concreto de peso ligero con las características aquí estudiadas; sin embargo, sí se debe incluir implícitamente el efecto de la baja resistencia de todos los tipos de concreto empleados. 
De acuerdo con las tendencias de los resultados experimentales, los efectos principales que se deben considerar para evaluar $\eta_{h}$, son: la distribución de deformaciones a lo largo de las diagonales, la cuantía de refuerzo en el alma del muro y el tipo de refuerzo utilizado para proveer dicha cuantía. En cuanto a la cuantía de refuerzo, Sánchez (2010) detectó que la eficiencia del refuerzo horizontal en trasmitir cortante se reduce sistemáticamente al incrementar su cuantía. Adicionalmente, Wood (1990) observó que la ecuación propuesta por el ACI-318 para diseño sísmico de muros, sobrestima la tasa de incremento de resistencia atribuible al refuerzo del alma; es decir, la eficiencia del refuerzo en el alma disminuye a medida que se aumenta la cuantía. La cuantías de refuerzo horizontal de los especímenes utilizados en los estudios de Sánchez (2010) y Wood (1990) variaron aproximadamente entre $0.12 \%$ y $1.4 \%$ y, entre 0.1 y $1.9 \%$, respectivamente. Sin embargo, para las cuantías de refuerzo nominales aquí utilizadas $(0.125 \%$ y $0.25 \%$ ), no se observó una tendencia clara de disminución de eficiencia al incrementar el valor de dicha cuantía. La distribución de deformaciones se refiere a la variación de la magnitud de esfuerzos cortantes en la altura del muro, lo cual genera que el ancho de las grietas inclinadas sea mínimo en las zonas cercanas a los extremos de las secciones del muro. Por consiguiente, en la mayoría de los casos, no es posible que todo el refuerzo transversal que cruza las grietas en la base o en el extremo superior del muro desarrolle la fluencia. En general, la fluencia del refuerzo horizontal se concentra en las barras/alambres colocados en la parte central del alma (media altura y media longitud). Este efecto se observó en los muros ensayados dinámicamente en mesa vibradora, así como en especímenes ensayados bajo carga lateral cíclica y monótona reportados por Leiva y Montaño (2001), Flores et al. (2007), Sánchez (2010), entre otros.

A pesar de las diferencias significativas entre el comportamiento esfuerzo-deformación de las barras corrugadas y los alambres de refuerzo, ninguna de las metodologías disponibles considera explícitamente el efecto del tipo de refuerzo en la eficiencia de refuerzo horizontal para contribuir a la resistencia a cortante. En primer lugar, el término "fluencia" se define para aceros de refuerzo en los cuales no se observa aumento de resistencia hasta tanto no se desarrolle una plataforma de fluencia bien definida [figura 3(b)]. En el caso de los alambres de las mallas, no existe un punto de fluencia específico y, por tanto, para alambres de malla se debe hacer referencia a "plastificación". Tomando en cuenta que la cuantía mínima de refuerzo se coloca para mantener la carga de agrietamiento por tensión diagonal, si se utiliza acero de refuerzo con mayor esfuerzo de fluencia, los reglamentos de diseño permiten la disminución de la cuantías de refuerzo, en función del incremento del esfuerzo de fluencia de los alambres de las mallas en comparación con el de las barras corrugadas de acero. En otras palabras, es necesario reconocer que en los alambres de las mallas no existe una plataforma de fluencia que retrase el aumento de su resistencia a tensión. Las Normas NTC-M (2004) consideran dicho efecto para el diseño sísmico de muros de mampostería reforzada. Sin embargo, el concepto anterior es aplicable a los alambres de malla que desarrollan ductilidad adecuada antes de presentarse la fractura; es decir, no se debe extrapolar de forma directa a los alambres de refuerzo con capacidad de ductilidad limitada (alargamiento). Por ejemplo, en los alambres aquí utilizados, el tramo comprendido entre el inicio de la "fluencia" y la capacidad máxima de deformación fue muy corto en comparación con las barras corrugadas [figura 3(b)], lo cual originó que se presentara la fractura del material con sólo un pequeño incremento de la deformación. Adicionalmente, el alargamiento promedio de estos alambres (1.9\%) no cumplió con la especificación de la Norma NMX-B-253 (2006), donde se estipula que el alargamiento mínimo debe ser igual a 6\%. Tal como se observó durante los ensayos de lo muros, una vez se fracturan los alambres se genera un mecanismo de falla súbito no deseado. De esta manera, para propósitos de diseño sísmico de muros reforzados utilizando alambres de malla con capacidad de ductilidad limitada, no se deben disminuir las cuantías de refuerzo y, al mismo tiempo, convendría utilizar parámetros de capacidad de ductilidad estructural asociados a factores de seguridad mayores que los recomendados para muros reforzados con barras corrugadas de acero (en la sección "parámetros para diseño sísmico" se recomiendan estos parámetros). 
En los ensayos dinámicos, la eficiencia medida del refuerzo horizontal del alma fue mayor en los muros con barras corrugadas reforzados con la cuantía mínima de refuerzo a cortante especificada en el NTC-C (2004), que en los muros reforzados con malla de alambre soldado y el 50\% de la cuantía mínima. En los dos tipos de muros, las eficiencias promedio medidas fueron iguales al $86 \%$ y $78 \%$, respectivamente; es decir, la disminución de eficiencia fue cercana al $10 \%$ para los muros con malla de alambre soldado (Carrillo, 2010a). Tomando en cuenta que la eficiencia del refuerzo horizontal podría aumentar al disminuir la cuantía de refuerzo y que el tipo de refuerzo no afecta de forma directa su valor numérico, el efecto de la distribución de deformaciones en los muros con malla de alambre soldado es mayor que en los muros con barras corrugadas. A partir de las observaciones y las tendencias de los resultados, se propone utilizar la ecuación 2 para calcular el factor de eficiencia $\eta_{h}$, la cual considera la distribución de deformaciones a lo largo de las diagonales, la cuantía de refuerzo en el alma del muro y el tipo de refuerzo utilizado para proveer dicha cuantía. Los valores aquí propuestos se indican en la figura 3(a) (“Este estudio"), junto con las metodologías de Leiva y Montaño (2001), Flores et al., 2007, Sánchez (2010) y los reglamentos NTC-C (2004), NTC-M (2004) y ACI-318 (2008). En la figura 3(a) también se incluyen los resultados medidos en los muros ensayados de forma dinámica.

$\eta_{h}=0.8 \quad$ Barras corrugadas

$\eta_{h}=0.7 \quad$ Malla alambre soldado

Los valores de la ecuación 2 se deben utilizar en muros con valores del producto $\rho_{h} f_{y h} \leq 1.25 \mathrm{MPa}$. El límite de la ecuación se estableció a partir de las características del refuerzo de los muros utilizados para la calibración del modelo. De acuerdo con la ecuación 2, todas las barras/alambres de refuerzo horizontal no alcanzan, en promedio, a fluir/plastificar al momento de alcanzar la resistencia del muro.
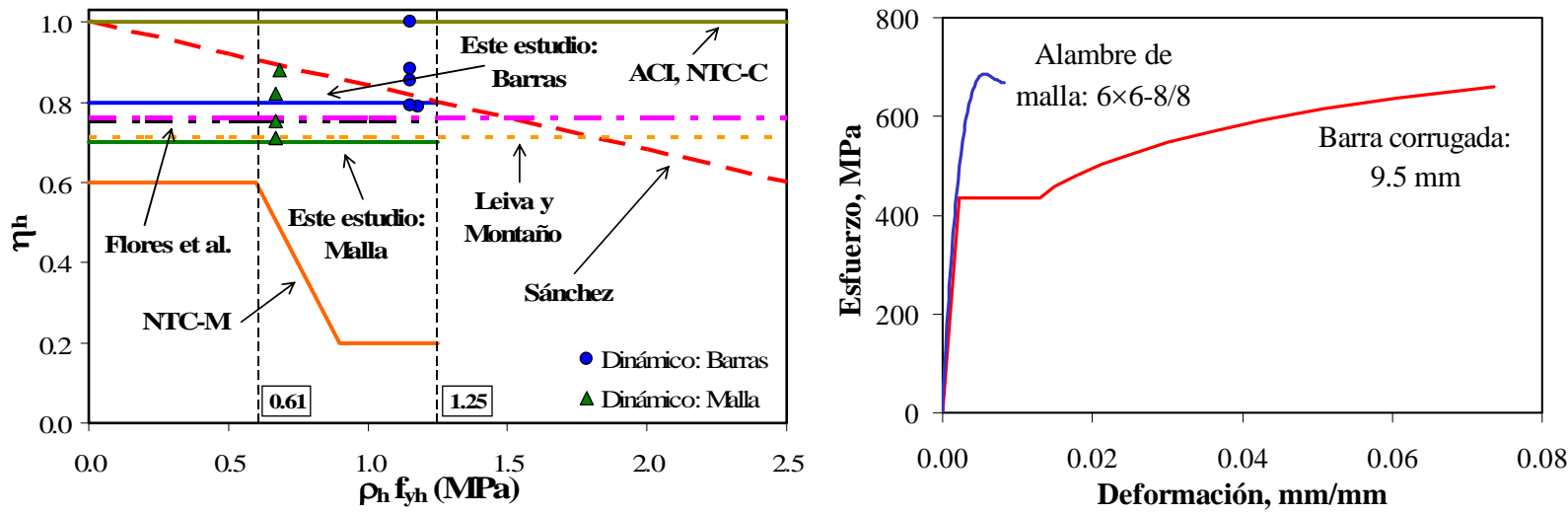

(a) Variación del factor de eficiencia

(b) Curvas características esfuerzo-deformación del acero

Figura 3 Factor de eficiencia del refuerzo horizontal

Para garantizar distribución uniforme de grietas diagonales, es necesario proporcionar una cuantía mínima de refuerzo vertical en el alma, la cual depende principalmente de la cuantía de refuerzo horizontal y de la relación de aspecto del muro. Con base en las deformaciones medidas en el acero de refuerzo vertical del alma del muro, se propone calcular dicha cuantía a partir la ecuación 3, cuyos resultados se ilustran en la figura 4 ("Este estudio"). 
$\rho_{v}=\rho_{\min }+0.5\left(2-\frac{H}{l_{w}}\right)\left(\rho_{h}-\rho_{\min }\right) \geq \rho_{\text {min }}$

La ecuación 3 proporciona resultados iguales a la ecuación propuesta en el capítulo 11 del ACI-318, cuando $\rho_{v}=0.0025$ y la constante numérica 2 se reemplaza por 2.5 . Si en la ecuación $3 \rho_{h}=\rho_{\text {min }}$, entonces $\rho_{v}=\rho_{\text {min }}$; de lo contrario $\rho_{v}$ disminuye gradualmente al aumentar $h_{w} / l_{w}$ hasta un valor igual a $\rho_{\text {min }}$. De acuerdo con la tendencia observada en los muros aquí estudiados (figura 4), $\rho_{v}$ podría ser aún menor; sin embargo, no se dispone de datos experimentales suficientes para proponer un factor de eficiencia del refuerzo vertical menor que el calculado por medio de la ecuación 3. Por ejemplo, en los muros para vivienda aquí estudiados, la cuantía de refuerzo vertical en el alma siempre fue igual a la cuantía horizontal.

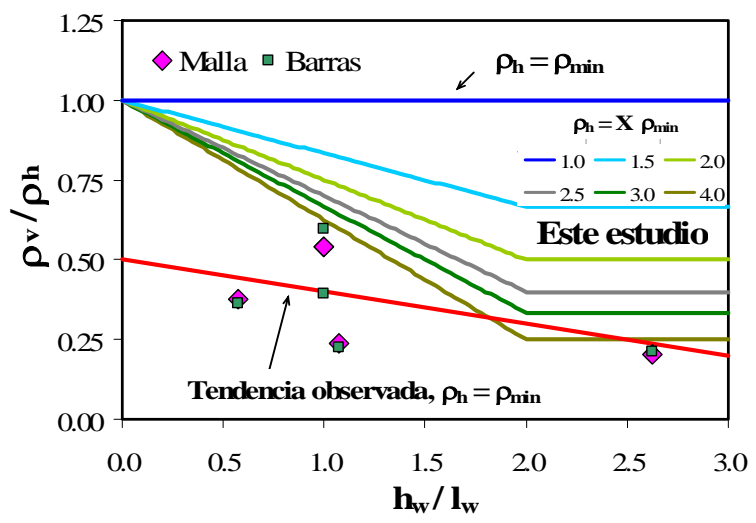

Figura 4 Requisitos del refuerzo vertical en el alma

En la ecuación 1, el factor $\alpha_{1}$ depende principalmente de: (a) la geometría y las condiciones de frontera del muro (cociente $M / V l_{w}$, cociente del momento de flexión y el producto de la fuerza cortante por la longitud del muro) y (b) el esfuerzo vertical axial que actúa sobre el muro $\left(\sigma_{v}\right)$. En la metodología de Sánchez (2010), el factor depende adicionalmente de la cuantía de refuerzo vertical a cortante en el alma del muro. En cuanto al cociente $M / V l_{w}$, se ha observado que los muros robustos (valores $M / V l_{w}$ bajos) desarrollan resistencias a cortante mayores que muros más altos con propiedades de materiales similares. En lo referente al esfuerzo axial, en los muros de concreto en viviendas de baja altura, el esfuerzo vertical axial de compresión en condiciones de servicio es relativamente bajo ( $\sigma_{v} \approx 0.25 \mathrm{MPa}$; Carrillo, 2010a). Sin embargo, ante una solicitación sísmica real, las aceleraciones verticales y/o el efecto de acoplamiento entre muros pueden originar que los esfuerzos verticales axiales de compresión se incrementen o se disminuyan, incluso alcanzando esfuerzos verticales axiales de tensión. Si se presentan esfuerzos de tensión, la capacidad a cortante del muro disminuirá. En ensayos cuasi-estáticos, este efecto es imperceptible, pues no existe fuerza inercial sobre la masa que se coloca sobre el muro para generar el esfuerzo vertical; es decir, el esfuerzo vertical siempre contribuye a la resistencia a cortante. Por tanto, para fines prácticos de diseño sísmico, la contribución del esfuerzo vertical axial a la resistencia a cortante del muro se incluyó utilizando $\sigma_{v}=0$; es decir, que la aceleración vertical sería aproximadamente equivalente a $1.0 \mathrm{~g}$. Adicionalmente, se considera que este enfoque es realista en zonas de amenaza sísmica alta, donde la aceleración vertical es importante, y conservador en zonas de amenaza sísmica baja, pues la aceleración vertical es relativamente baja con respecto a la aceleración horizontal.

En la ecuación 1, el límite superior representa la resistencia que puede desarrollar el muro antes de que se origine falla por compresión diagonal. En este límite, el factor $\alpha_{2}$ relaciona la contribución máxima 
del concreto a la resistencia al cortante por compresión diagonal y la raíz cuadrada de la resistencia a la compresión del concreto. Las fallas por compresión diagonal se presentan principalmente en: a) muros con altas cuantías de refuerzo a cortante, las cuales impiden que el refuerzo experimente deformaciones plásticas y, b) muros con resistencias a la compresión del concreto bajas o en muros de concreto agrietados. A partir de las tendencias observadas de los resultados experimentales, para propósitos de diseño sísmico de viviendas con las características estudiadas, se propone calcular los factores $\alpha_{1}$ y $\alpha_{1}$ por medio de la ecuación 4. Los valores aquí propuestos se indican en la figura 5 ("Este estudio"), junto con las metodologías de otros estudios y reglamentos.

$$
\begin{aligned}
& \alpha_{1}=0.21-0.02\left(\frac{M}{V l_{w}}\right) \quad(\mathrm{MPa}) \\
& \alpha_{2}=0.44-0.02\left(\frac{M}{V l_{w}}\right) \quad(\mathrm{MPa})
\end{aligned}
$$

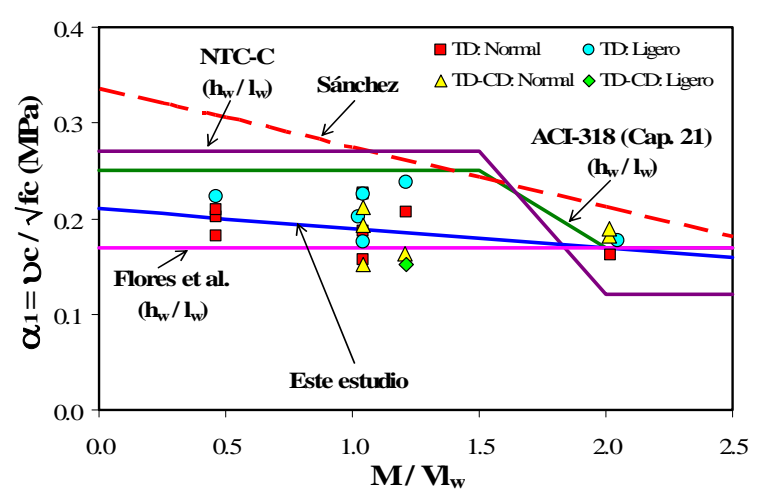

(a) Tensión diagonal $\left(\alpha_{1}\right)$

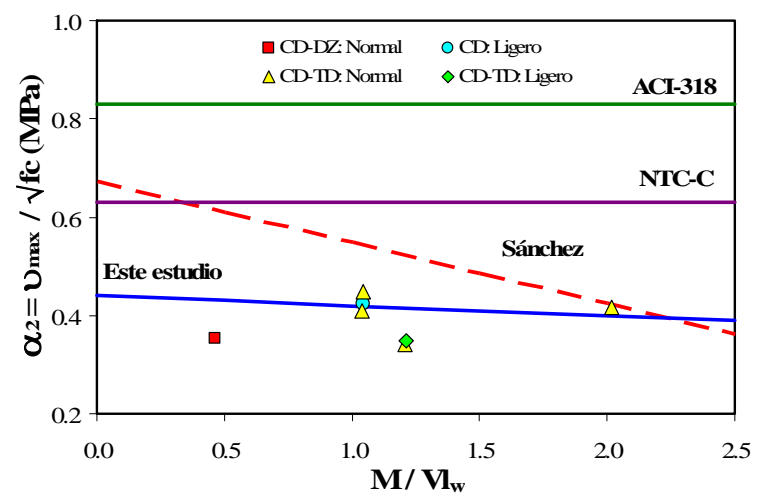

(b) Compresión diagonal $\left(\alpha_{2}\right)$

Figura 5 Contribución del concreto a la resistencia al cortante

\section{Deslizamiento}

Para los muros de las características estudiadas, se plantea incluir el factor $\psi$ en las ecuaciones de la sección 2.5.10 de las NTC-C (2004). Por tanto, se propone que la resistencia al cortante por fricción esté asociada al menor de los valores calculados con las ecuaciones 5 a 7 . En dichas ecuaciones, $A$ es el área de la sección definida por el plano crítico, $A_{v f}$ es el área total de refuerzo de cortante por fricción, $f_{y}$ es el esfuerzo especificado de fluencia del acero de cortante por fricción (para el diseño, $f_{y} \leq 412 \mathrm{MPa}$ ), $N_{u}$ es la fuerza de diseño de compresión normal al plano crítico, $\mu$ es el coeficiente de fricción que se toma como 1.4 para concreto colado monolíticamente, 1.0 para concreto colado contra concreto endurecido y, $\psi$ representa la eficiencia del refuerzo a cortante por fricción y es igual a 0.45.

$V_{n}=\mu\left(\psi A_{v f} f_{y}+N_{u}\right)$

$V_{n}=\left[1.4 A+0.8\left(\psi A_{v f} f_{y}+N_{u}\right)\right] \quad(\mathrm{MPa})$

$V_{n}=0.25 f_{c}^{\prime} A$ 
El factor $\psi$ se calculó a partir de los resultados medidos en los dos muros robustos $\left(h_{w} / l_{w}=0.5\right)$ en los cuales se observó este tipo de falla. De forma similar al refuerzo horizontal en el alma del muro, el factor $\psi$ tiene en cuenta que no todo el refuerzo que cruza la grieta de cortante alcanza la fluencia, especialmente en muros largos o muy largos. Probablemente, el fenómeno se genera porque la separación vertical del bloque que se desliza no es constante a lo largo del plano de deslizamiento. Aunque no se utilizaron muchos muros para calibrar el factor $\psi$, se trató de recoger la tendencia observada. No obstante, es claro que se necesitan más ensayos y estudios que incluyan el efecto de la cuantía y el tipo de acero de refuerzo de cortante por fricción, el tipo de concreto, la relación de aspecto y el esfuerzo axial, en la resistencia a cortante por fricción de muros de concreto con las características aquí estudiadas.

\section{Estimación del cociente $M / V I_{w}$}

Las ecuaciones del modelo de predicción aquí propuesto dependen del cociente $M / V l_{w}$; es decir, el cociente entre el momento de flexión $(M)$ y el producto de la fuerza cortante por la longitud del muro $\left(V l_{w}\right)$. Si en muros ensayados en voladizo la carga se aplica a una altura igual a la altura del muro $\left(h_{w}\right)$, la relación de aspecto $\left(h_{w} / l_{w}\right)$ es igual al cociente $M / V l_{w}$ Sin embargo, en muros con nivel de acoplamiento común, $h_{w} / l_{w}$ es usualmente mayor que $M / V l_{w}$. Para el diseño sísmico de viviendas de baja altura, $M$ y $V$ representan el momento y el cortante de diseño en la base del muro. Por tanto, el valor del cociente $M / V l_{w}$ se debe obtener utilizando los parámetros calculados durante el proceso de diseño sísmico. A partir de los resultados del análisis y diseño de ocho prototipos de viviendas de uno y dos niveles ubicados en diferentes zonas de la República Mexicana (Carrillo, 2010a), se calculó el valor del cociente $M / V l_{w}$ asociado a cada muro con relación de aspecto conocida $\left(h_{w} / l_{w}\right)$. En la figura 6 se indica cada pareja de datos para las viviendas de uno (Analítico-1N) y dos niveles (Analítico-2N) y, se incluye la propuesta de Sánchez (2010). En la figura se observa que no existe una diferencia evidente entre las tendencias para muros en viviendas de uno y dos niveles.

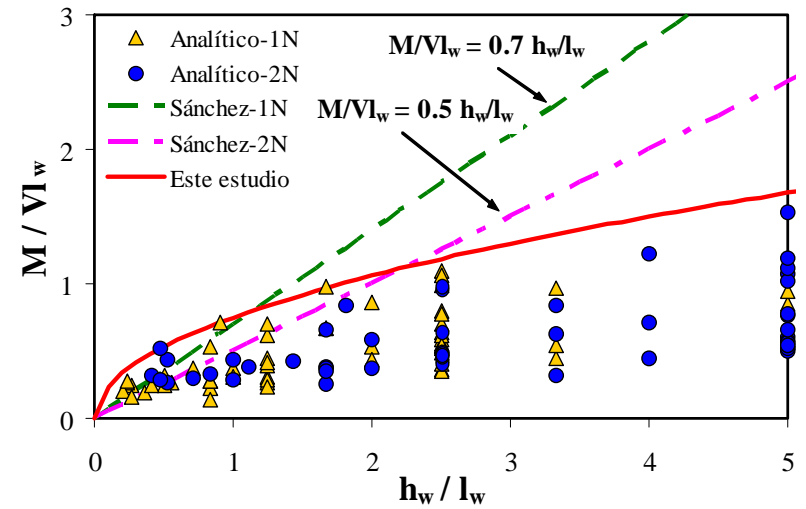

Figura 6 Relación entre $M / V l_{w}$ y $h_{w} / l_{w}$ para muros continuos en viviendas de uno (1N) y dos (2N) niveles

A medida que disminuye el cociente $M / V l_{w}$, aumenta la capacidad de resistencia a cortante del muro. Por consiguiente, para propósitos de diseño sísmico reglamentario, es deseable tener una expresión que estime de forma conservadora el cociente $M / V l_{w}$ a partir de la relación $h_{w} / l_{w}$. De esta manera, para muros continuos en viviendas de uno y dos niveles, se propone calcular el cociente $M / V l_{w}$ por medio de la ecuación 8, la cual se obtuvo a partir de un análisis de regresión no lineal y cuyos resultados se muestran en la figura 6 ("Este estudio"). No obstante, siempre es deseable calcular el cociente $M / V l_{w}$ a partir de los resultados de diseño $(M$ y $V)$. 
$\left(\frac{M}{V l_{w}}\right)=0.75 \sqrt{\frac{h_{w}}{l_{w}}}$

Para evaluar la bondad del modelo de resistencia a cortante, se llevó a cabo un análisis estadístico de los cocientes entre las resistencias calculadas y experimentales para los muros ensayados de forma dinámica y bajo carga lateral cíclica (Carrillo, 2010a). Dicho análisis demostró que las resistencias predichas son muy similares a las medidas y que la dispersión es menor que en los modelos disponibles. Por tanto, se considera que metodología aquí propuesta es adecuada para fines de diseño sísmico reglamentario de muros de concreto para vivienda de baja altura.

\section{PARÁMETROS PARA DISEÑO SÍSMICO}

Para diseño sísmico de estructuras, en el Distrito Federal de México es obligatoria la utilización de las Normas Técnicas Complementarias para Diseño por Sismo (NTC-S, 2004). En el resto del país, la mayoría de los estados adopta el Manual de Diseño de Obras Civiles de la Comisión Federal de Electricidad (MDOC-CFE, 1993, 2008). Sin embargo, en el cuerpo principal de las NTC-S (2004), las ordenadas espectrales no representan demandas elásticas, ya que fueron reducidas con fines de simplificación por un factor de "sobrerrestencia” $(R)$ cuyo valor máximo es igual a 2.5 (Rosenblueth et al., 1991). Un enfoque similar se utiliza en la versión del año 1993 del MDOC-CFE. En el apéndice A de las NTC-S (2004) y en la versión del año 2008 del MDOC-CFE, las ordenadas espectrales sí representan demandas elásticas. Por tanto, algunos parámetros de diseño que se estipulan en el apéndice A de las NTC-S (2004) y en el MDOC-CFE (2008), por ejemplo los factores $Q$ y $Q$ ', se relacionan pero no tienen el mismo significado que los especificados en el cuerpo principal de las NTC-S (2004) o en el MDOCCFE (1993). Con el propósito de aplicar adecuadamente los conceptos en el proceso de diseño e incluir por separado los parámetros que controlan la respuesta de las estructuras, para diseño sísmico se recomienda utilizar sólo las metodologías del apéndice A de las NTC-S (2004) o del MDOC-CFE (2008), cuya representación se indica en el diagrama cortante basal versus desplazamiento de azotea $(V-\Delta)$ que se muestra en la figura 7(a). En este artículo, los resultados se presentan siguiendo los lineamientos de estas metodologías.

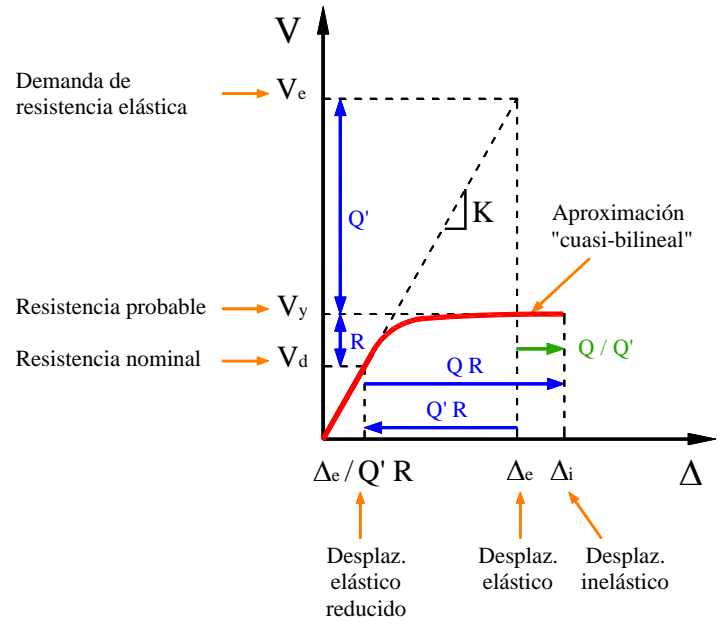

(a) Metodología de diseño sísmico

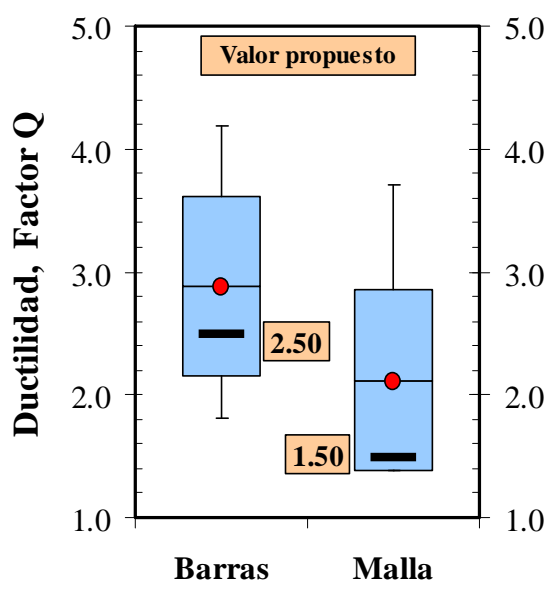

(b) Factor de comportamiento sísmico, Q

Figura 7 Diseño sísmico con fines reglamentarios 


\section{Factor de comportamiento sísmico}

Por razones básicamente económicas, es impráctico pretender que las estructuras resistan los grandes temblores sin rebasar su intervalo de comportamiento elástico. Por tanto, los criterios vigentes de diseño admiten que la estructura experimente incursiones en el intervalo no-lineal ante el sismo que caracteriza el estado límite de colapso. Esto permite limitar las demandas de fuerza en los elementos estructurales (utilizar resistencias de diseño menores), a cambio de que se presenten demandas de ductilidad limitadas y cierto nivel de daños provocados por la fluencia de algunas secciones de la estructura. En la mayoría de los reglamentos, las fuerzas sísmicas de diseño se reducen en función de la capacidad de ductilidad de las estructuras, utilizando el factor de reducción por ductilidad que se denota como Q' [figura 7(a)]. Sin embargo, no todo el exceso de la capacidad estructural real ante sismo con respecto a la calculada se debe a comportamiento dúctil. La mayoría de las estructuras cuenta con reservas extras de capacidad ante carga sísmica no consideradas en el diseño convencional, las cuales hacen que la estructura tenga una sobrerresistencia (MDOC-CFE, 2008). La opción más práctica de tomar en cuenta este efecto, consiste en aplicar un factor reductor, $R$, del lado de las acciones.

El factor de reducción por ductilidad Q', se define como la máxima disminución de resistencia que se puede usar para mantener la demanda de ductilidad de desplazamiento, por debajo de la capacidad máxima de ductilidad que se le puede exigir al sistema, $\mu_{\text {cap }}$; es decir, si se utiliza un factor de reducción por ductilidad mayor, entonces la demanda de ductilidad excederá la capacidad máxima de ductilidad. En el apéndice A de las NTC-S (2004) y en MDOC-CFE (2008), $\mu_{\text {cap }}$ se denota por medio de $Q$ haciendo referencia al factor de comportamiento sísmico [figura 7(a)]. Para cada uno de los especímenes ensayados de forma dinámica y bajo carga lateral cíclica se calculó la capacidad máxima de ductilidad, $\mu_{\text {cap }}$, definida como el cociente entre el desplazamiento último y el desplazamiento de fluencia. El desplazamiento último debe estar relacionado con límites tolerables de capacidad de resistencia y disipación de energía y, por tanto, en los especímenes estudiados se midió el desplazamiento asociado a una disminución de resistencia del $20 \%$ respecto a la máxima alcanzada. De esta manera, el factor de comportamiento sísmico $Q$ en realidad no sólo está asociado a la ductilidad estructural, sino también al deterioro o efecto que puede llegar a contrarrestar gran parte de la capacidad extra en resistencia que suministra la ductilidad y a reservas de capacidad ante carga sísmica que los métodos convencionales de diseño no consideran (MDOC-CFE, 2008).

Al procesar los resultados se observó que las características principales que afectan el factor de ductilidad son el tipo de refuerzo a cortante en el alma de los muros y la cociente $M / V l_{w}$. No se observaron diferencias significativas entre muros con concretos de peso normal, de peso ligero y autocompactable. Sin embargo, si para propósitos de diseño de viviendas se recomienda calcular la ductilidad en función del cociente $M / V l_{w}$ o $h_{w} / l_{w}$, sería difícil establecer un solo valor de la capacidad máxima de ductilidad para el diseño sísmico de toda la vivienda, ya que en la estructura se encuentran muros con diferentes geometrías. Por tanto, para fines prácticos de diseño reglamentario, los datos fueron agrupados sólo en dos categorías: muros con barras corrugadas y muros con malla de alambre soldado. En la figura 7(b) se muestran gráficamente los parámetros estadísticos del factor del comportamiento sísmico $(Q)$ para los especímenes ensayados (el círculo al centro del recuadro indica el valor promedio y la altura total del recuadro representa dos veces la desviación estándar). Tal como se esperaba, para especímenes controlados por cortante, los valores de la capacidad máxima de ductilidad son bajos, especialmente para los muros reforzados con malla de alambre soldado. En estos muros, la capacidad máxima de desplazamiento se alcanzó para una distorsión sólo un poco mayor que la asociada a la resistencia. A pesar de que los muros reforzados con barras corrugadas incursionaron apreciablemente en el intervalo inelástico, la tasa elevada de degradación de resistencia originó que los desplazamientos asociados a una disminución de resistencia del $20 \%$ fueran relativamente bajos. Como se observa en la figura 7(b), el valor promedio fue igual a 2.1 para muros reforzados en el alma con malla de alambre soldado y 2.9 para muros con barras corrugadas. 
Tomando en cuenta que la muestra analizada corresponde a muros con diferentes geometrías (robustos, cuadrados y esbeltos), de forma similar a lo que se tendría en una vivienda real, la dispersión de los resultados es alta.

En la tabla 4 se muestra que en las Normas NTC (2004) y en MDOC-CFE (2008) no se especifica explícitamente el factor de comportamiento sísmico para muros de concreto en viviendas de baja altura (asociado al nivel de desempeño de seguridad al colapso), pues se supone que los valores propuestos para muros de edificios son aceptables para viviendas de baja altura. Por tanto, tomando en cuenta que para el diseño sísmico de toda la vivienda se utiliza un solo valor de la capacidad máxima de ductilidad, en este estudio se recomienda utilizar un valor de $Q$ igual a 2.5 para muros reforzados con barras corrugadas y 1.5 para muros con malla de alambre soldado. Los valores recomendados también se indican en la gráfica de la figura 7(b), por medio de una barra horizontal más gruesa. El valor recomendado para muros reforzados con barras corrugadas $(Q=2.5)$ corresponde al percentil 42; es decir, el $58 \%$ de los datos se encuentran por encima de este valor. En cambio, para los muros reforzados con malla de alambre soldado $(Q=1.5)$ corresponde al percentil 7; es decir, el 93\% de los datos se encuentran por encima del valor recomendado. Estos porcentajes reflejan, de una forma indirecta, el factor de seguridad intrínseco en los dos tipos de refuerzo utilizado en los muros; es decir, el factor de seguridad es mayor en los muros reforzados con malla de alambre soldado donde se observó un modo de falla frágil caracterizado por la plastificación, incipiente en el mejor de los casos, y posterior fractura de los alambres que exhibieron capacidad de ductilidad limitada.

Tabla 4 Valores de $Q$ y $R_{P}$ estipulados en NTC (2004) y MDOC-CFE (2008)

\begin{tabular}{|c|c|c|c|}
\hline $\begin{array}{c}\text { Ocupación } \\
\text { inmediata (OI) }\end{array}$ & \multicolumn{2}{|c|}{$\begin{array}{l}\text { Seguridad al } \\
\text { colapso (SC) }\end{array}$} & \multirow[t]{2}{*}{ Sistema estructural } \\
\hline$R_{P(\mathrm{OI})}(\%)$ & $Q$ & $R_{P(S C)}(\%)$ & \\
\hline \multirow{8}{*}{0.2 o $0.4^{(1)}$} & 3.0 & 1.50 & $\begin{array}{l}\text { Muros de concreto reforzado que son capaces de resistir al menos el } \\
80 \% \text { de las fuerzas laterales totales y que cumplen los requisitos para } \\
\text { muros dúctiles }\end{array}$ \\
\hline & \multirow{4}{*}{2.0} & 1.00 & $\begin{array}{l}\text { Muros de concreto reforzado en los cuales los elementos de borde no } \\
\text { cumplen los requisitos de ductilidad }\end{array}$ \\
\hline & & 0.50 & Muros de mampostería de piezas \\
\hline & & 0.40 & $\begin{array}{l}\text { macizas, conimada por castillos y } \\
\text { dalas de concreto reforzado }\end{array}$ \\
\hline & & 0.40 & $\begin{array}{l}\text { Muros de mampostería de piezas multiperforadas, con al menos la } \\
\text { cuantía mínima de refuerzo horizontal y confinada con castillos } \\
\text { exteriores }\end{array}$ \\
\hline & \multirow{2}{*}{1.5} & 0.20 & $\begin{array}{l}\text { Muros de mampostería de piezas huecas, confinada o con refuerzo } \\
\text { interior }\end{array}$ \\
\hline & & 0.20 & $\begin{array}{l}\text { Muros de mampostería reforzada interiormente con barras o alambres } \\
\text { corrugados de acero, horizontales y verticales }\end{array}$ \\
\hline & 1.0 & 0.15 & Muros de mampostería no reforzada, ni confinada \\
\hline
\end{tabular}

${ }^{(1)}$ Cuando no existan elementos incapaces de soportar deformaciones apreciables, como los muros de mampostería, o que éstos estén separados de la estructura principal de manera que no sufran daños por sus deformaciones.

Como se muestra en la tabla 4, el valor aquí recomendado para muros de concreto reforzados en el alma con barras corrugadas $(Q=2.5)$ es ligeramente menor que el valor especificado en NTC-C (2004) para muros que cumplen los requisitos para muros dúctiles $(Q=3.0)$, y es un poco mayor que el valor estipulado en NTC-M (2004) para muros de mampostería de piezas macizas confinada con castillos y dalas, o para muros de mampostería reforzada de piezas multiperforadas y confinada con castillos exteriores $(Q=2.0)$. Para muros de concreto reforzados en el alma con malla de alambre soldado, el valor 
aquí recomendado $(Q=1.5)$ es menor que el especificado en NTC-C para muros de concreto que no cumplen los requisitos para muros dúctiles $(Q=2.0)$, y es igual al valor estipulado en NTC-M para muros de mampostería de piezas huecas confinada o reforzada, o para muros de mampostería reforzada interiormente.

\section{Distorsiones permisibles de entrepiso}

En los últimos años se ha intensificado la reevaluación de los criterios fundamentales del diseño sísmico. Uno de los avances principales ha sido el planteamiento de la ingeniería sísmica basada en desempeño, la cual se refiere a la selección de "objetivos de desempeño sísmico" definidos como "la relación entre el nivel de desempeño esperado con los niveles esperados de movimiento sísmico del suelo" (Priestley, 2000). Un nivel de desempeño representa básicamente una banda distinta en el espectro de daño de los elementos estructurales y no estructurales. Los niveles de desempeño son introducidos como valores límite de indicadores de desempeño medibles de la respuesta estructural, tales como distorsión, ductilidad, anchura de grietas, índices de daño estructural, entre otros (Guljas y Sigmund, 2006). Cuando se seleccionan los niveles de desempeño, los valores límite asociados (variables de decisión) se convierten en el criterio de aceptación que se debe verificar en las etapas posteriores del diseño. Los indicadores de desempeño o variables de decisión no son definidas por el diseñador, ya que éstas se especifican en el reglamento que está siendo utilizado.

El daño sufrido por una estructura mientras disipa energía durante un sismo es dependiente de los desplazamientos inelásticos que la estructura experimenta. Por tanto, los objetivos de desempeño estructural definidos en términos de límites de distorsión pueden ser relacionados de forma directa con el daño. Sin embargo, la definición de límites confiables y realistas de distorsión que estén asociados a estados de daño conocidos, continúa siendo uno de los temas primordiales sin resolver en los procedimientos de diseño sísmico basados en desempeño. De acuerdo con Ghobarah (2004), los límites de distorsión disponibles son conservadores para estructuras con comportamiento dúctil, pero son inseguros para estructuras con comportamiento poco dúctil. Del mismo modo, Duffey et al. (1994a, 1994b) señalan que las distorsiones permisibles especificadas en la mayoría de los reglamentos de diseño son generalmente poco conservadoras para muros de concreto con baja relación de aspecto ya que, de modo similar a las expresiones para calcular el periodo natural de vibración, las distorsiones de dichos reglamentos están dirigidas a muros de gran altura más que a muros bajos.

Con el propósito de evaluar el daño en los muros de concreto en viviendas de baja altura, cuyo comportamiento está gobernado por cortante, los indicadores de desempeño se establecieron con base en límites de distorsión permisible de entrepiso $\left(R_{P}\right)$. Para establecer los valores $R_{P}$, inicialmente se implantaron las resistencias de diseño para cada nivel de desempeño. Para DSBD de muros de concreto con las características particulares de viviendas de baja altura se propone seleccionar tres niveles de desempeño sísmico o estados de daño (tabla 5): a) ocupación inmediata (OI), b) protección de la vida (PV) y, c) seguridad al colapso (SC). Los niveles de desempeño de OI y PV están asociados al desarrollo del $25 \%$ y $75 \%$, respectivamente, de la resistencia $\left(0.25 V_{\max }\right.$ y $\left.0.75 V_{\max }\right)$. El nivel de desempeño de SC está asociado al desarrollo de la resistencia a cortante del muro $\left(V_{\max }\right)$ (Carrillo, 2010a). Como se muestra en la tabla 5, en este caso, los valores seleccionados son iguales a los propuestos por Sánchez (2010). Luego se establecieron los valores límite de distorsión total asociados a las resistencias de diseño definidas. Los valores se determinaron utilizando las curvas de histéresis medidas en los ensayos dinámicos y cuasiestáticos. En seguida se estableció de forma cualitativa el daño esperado, a partir del daño observado durante los ensayos dinámicos de los muros.

En las figuras 8 y 9 se presentan curvas de histéresis características para muros con refuerzo a cortante en el alma utilizando barras corrugadas y malla de alambre soldado, respectivamente. En las 
figuras se muestran por separado las curvas características para muros cuadrados, robustos y esbeltos. En la primera fila de la figura 8 se muestran las curvas para muros con el 50\% de la cuantía mínima a cortante estipulada en NTC-C (2004) y, en la segunda fila, para los muros con la cuantía mínima. Cuando se determinó el valor de $R_{P}$ para cada uno de los muros ensayados bajo carga lateral cíclica y de forma dinámica, se observó que el tipo de refuerzo a cortante en el alma y el cociente $M / V l_{w}$ o la relación $h_{w} / l_{w}$ son las características que mayor afectan el valor de $R_{P}$ (no se observaron diferencias significativas entre muros con concretos de peso normal, de peso ligero y autocompactable). Por ejemplo, para muros esbeltos se podrían plantear valores mayores que los propuestos para muros cuadrados o robustos (figuras 8 y 9). Sin embargo, para fines de diseño reglamentario no sería práctico proponer valores de $R_{P}$ o de $Q$ en función de $M / V l_{w}$ o $h_{w} / l_{w}$, tomando en cuenta que se debe utilizar un solo valor para el diseño de toda la vivienda, pues todos los muros de un entrepiso están acoplados por la losa de entrepiso y, por tanto, sujetos prácticamente al mismo valor de distorsión.

Tabla 5 Indicadores de desempeño propuestos para muros de concreto de vivienda

\begin{tabular}{|c|c|c|c|}
\hline $\begin{array}{c}\text { Nivel de } \\
\text { desempeño }^{(1)}\end{array}$ & $\begin{array}{l}\text { Resistencia } \\
\text { de diseño }\end{array}$ & $\begin{array}{l}\text { Distorsión total } \\
\text { límite, } R_{P}(\%)\end{array}$ & Daño esperado \\
\hline OI & $0.25 V_{\max }$ & $\begin{array}{l}<0.15, \text { Barras } \\
<0.10, \text { Malla } \\
<0.05 \text {, Sin ref. }\end{array}$ & $\begin{array}{l}\text { Daño leve: } \\
\text { Agrietamos por flexión en los extremos y grietas inclinadas } \\
\text { menores. }\end{array}$ \\
\hline PV & $0.75 V_{\max }$ & $\begin{array}{l}<0.40, \text { Barras } \\
<0.25 \text {, Malla } \\
<0.10, \text { Sin ref. }\end{array}$ & $\begin{array}{l}\text { Daño moderado: } \\
\text { Prolongación de las grietas inclinadas hasta los extremos del } \\
\text { alma, pero sin penetrar en los elementos de borde. }\end{array}$ \\
\hline SC & $V_{\max }$ & $\begin{array}{l}<0.65 \text {, Barras } \\
<0.35 \text {, Malla } \\
<0.15 \text {, Sin ref. }\end{array}$ & $\begin{array}{l}\text { Daño significativo: } \\
\text { Agrietamiento diagonal significativo y/o fluencia de algunas } \\
\text { barras/alambres en el alma del muro. Aplastamiento moderado } \\
\text { del concreto en el alma y daño alrededor de aberturas. }\end{array}$ \\
\hline
\end{tabular}

${ }^{(1)} \mathrm{OI}=$ Ocupación inmediata, $\mathrm{PV}=$ Protección de la vida, $\mathrm{SC}=$ Seguridad al colapso, ${ }^{(2)}$ Muros sin refuerzo a cortante en el alma (ver sección siguiente).

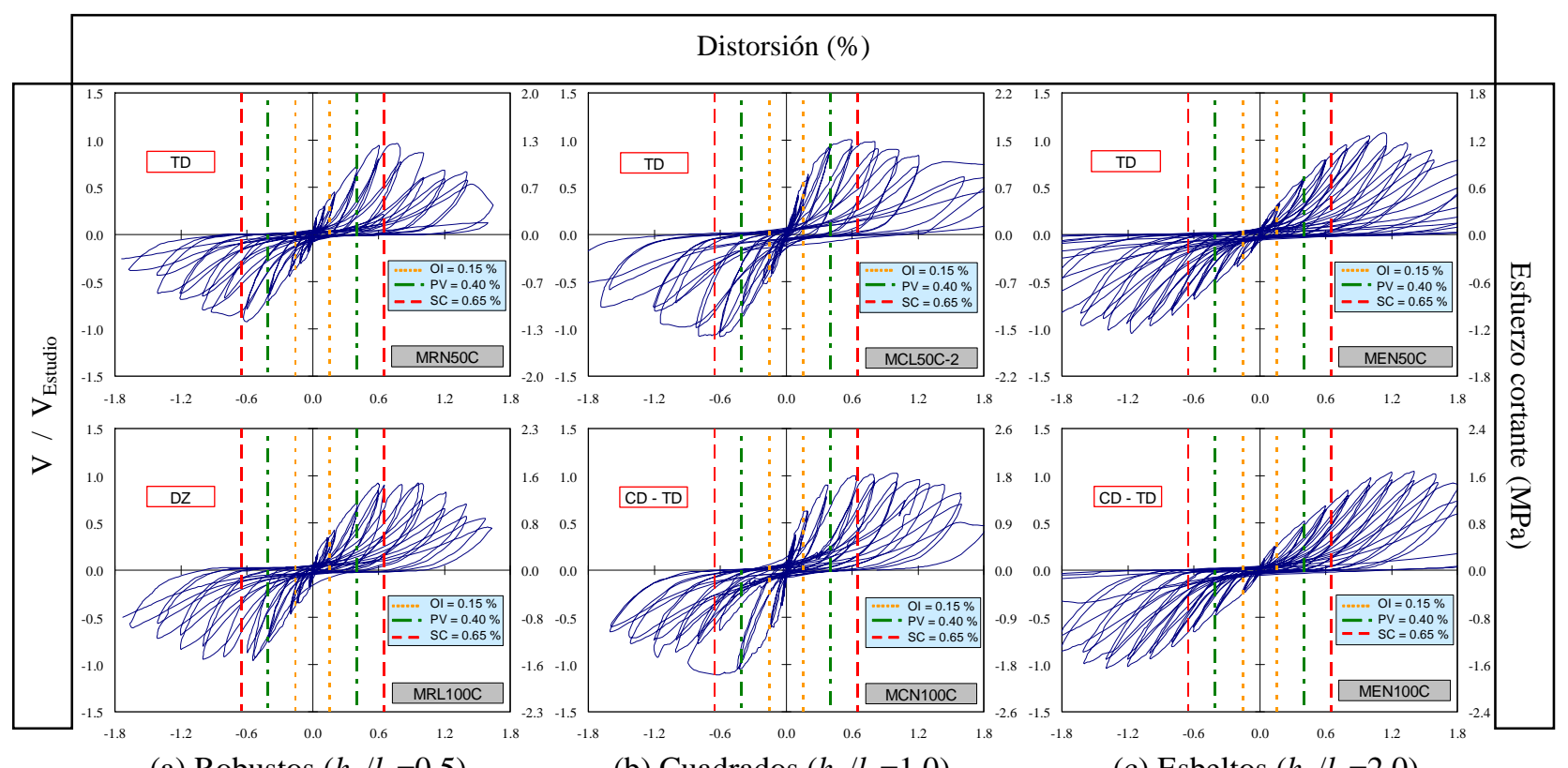

(a) Robustos $\left(h_{w} / l_{w}=0.5\right)$

(b) Cuadrados $\left(h_{w} / l_{w}=1.0\right)$

(c) Esbeltos $\left(h_{w} / l_{w}=2.0\right)$

Figura 8 Distorsiones permisibles en muros con barras corrugadas 
A partir de las curvas de histéresis medidas, para los tres niveles de desempeño definidos se proponen los valores límite de distorsión que se indican en la tabla 5, los cuales dependen del tipo de refuerzo a cortante en el alma. Los valores propuestos se muestran en las figuras 8 y 9. Se estableció un solo valor para muros con barras corrugadas, ya que no se observaron diferencias significativas entre los muros con las dos cuantías de refuerzo a cortante estudiadas. Como se observa en la tabla 4, para el nivel de desempeño de seguridad al colapso, el valor aquí recomendado para muros de concreto reforzados en el alma con barras corrugadas $\left(R_{P(S C)}=0.65 \%\right)$ es menor que el valor especificado en NTC-C (2004) para muros que no cumplen los requisitos para muros dúctiles $\left(R_{P(S C)}=1.0 \%\right)$, y es un poco mayor que el valor estipulado en NTC-M (2004) para muros de mampostería de piezas macizas confinada con castillos y dalas, con refuerzo horizontal o malla $\left(R_{P(S C)}=0.50 \%\right)$. Para muros de concreto reforzados en el alma con malla de alambre soldado, el valor aquí recomendado $\left(R_{P(S C)}=0.35 \%\right)$ es un poco menor que el valor estipulado en NTC-M para muros de mampostería reforzada de piezas multiperforadas confinada con castillos exteriores $\left(R_{P(S C)}=0.40 \%\right)$. Para el nivel de desempeño de ocupación inmediata, NTC (2004) y MDOC-CFE (2008) especifican dos valores de $R_{P(O I)}$ sin tomar en cuenta de forma directa el tipo de sistema estructural; es decir, los valores dependen exclusivamente de la presencia de elementos incapaces de soportar deformaciones apreciables $(0.20 \%$ o $0.40 \%$, tabla 4$)$. Los valores aquí propuestos son menores que los estipulados en dichos reglamentos; es decir, $R_{P(O I)}=0.10 \%$ para muros de concreto reforzados en el alma con malla de alambre soldado y $R_{P(O I)}=0.15 \%$ para muros reforzados con barras corrugadas.

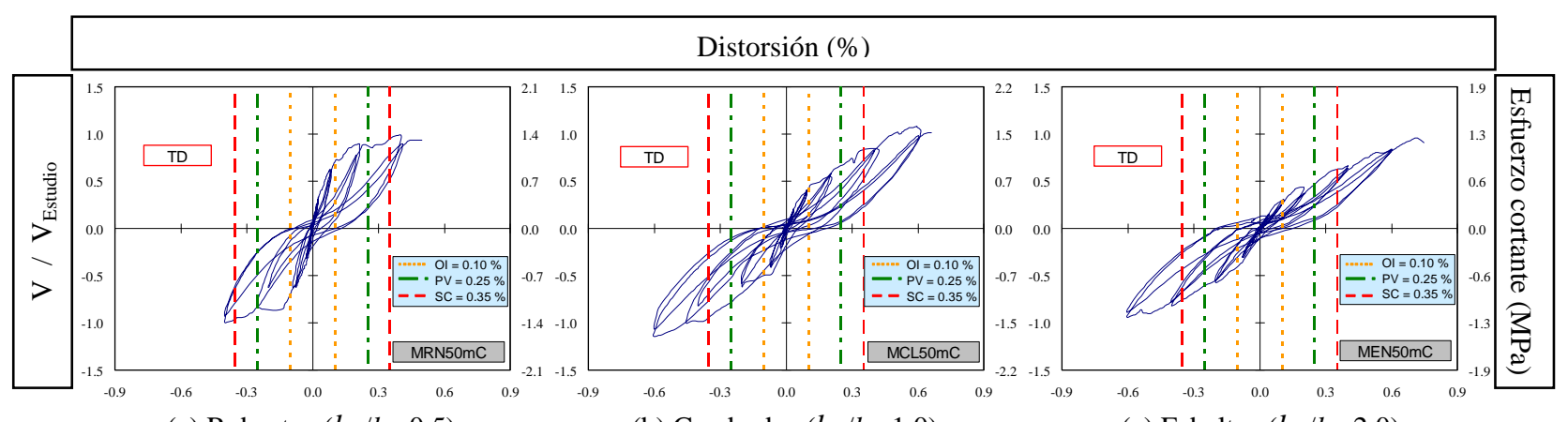

(a) Robustos $\left(h_{w} / l_{w}=0.5\right)$

(b) Cuadrados $\left(h_{w} / l_{w}=1.0\right)$

(c) Esbeltos $\left(h_{w} / l_{w}=2.0\right)$

Figura 9 Distorsiones permisibles en muros con malla de alambre soldado

\section{Comparación entre la demanda y la capacidad de resistencia de viviendas}

En esta sección se compara la demanda y la capacidad resistente de viviendas en términos del coeficiente sísmico $\left(C_{s}\right)$, el cual se emplea para representar la demanda sísmica de resistencia para fines de diseño reglamentario. La capacidad se determinó a partir de los resultados medidos en los ensayos dinámicos en mesa vibradora (Carrillo, 2010a). En la tabla 6 se presentan los coeficientes sísmicos medidos en los modelos, para tres estados límite: agrietamiento $(a)$, resistencia $(m)$ y último $(u)$. En la tabla se muestran, por separado, los resultados para los muros reforzados con malla de alambre soldado y para los muros reforzados con barras corrugadas. Para extrapolar los valores al prototipo se aplicó el factor de similitud de aceleración, modificado a partir de las relaciones de masa en el modelo y en el prototipo (Carrillo, 2010a). Además, se calculó el promedio de los coeficientes sísmicos en el prototipo para cada uno de los estados límite, de acuerdo con el tipo de refuerzo en el alma de los muros. A pesar de que los muros con malla de alambre soldado fueron reforzados con $50 \%$ de la cuantía mínima de refuerzo a cortante estipulada en las NTC-C (2004) y, los muros con barras corrugadas con el 100\% de dicha cuantía, los valores del coeficiente sísmico en el prototipo fueron notablemente similares (tabla 6). 
Para calcular las demandas de resistencia en viviendas ubicadas en diferentes zonas de la República Mexicana, se utilizaron las solicitaciones de diseño especificadas en el MDOC-CFE (2008) y los factores de comportamiento sísmico aquí recomendados $(Q=2.5$ para muros reforzados con barras corrugadas y $Q=1.5$ para muros con malla de alambre soldado). Se eligieron características del suelo que pudieran inducir las mayores demandas de resistencia y desplazamiento sobre las viviendas estudiadas. No se incluyó el factor de sobrerresistencia $(R=1.0)$, ya que la comparación se realizó utilizando resultados experimentales. No obstante, se podría utilizar un factor de sobrerresistencia un poco mayor que uno, ya que aunque los resultados experimentales se obtuvieron a partir del ensayo de muros aislados, la redundancia estructural, el acoplamiento muro-muro y muro-losa en la vivienda, contribuyen al efecto de la sobrerresistencia. Con el propósito de incluir exclusivamente el comportamiento de la vivienda, no se tuvo en cuenta el efecto de la interacción suelo-estructura. Los resultados fueron derivados para una vivienda de baja altura representada por un periodo fundamental de vibración, $T$, igual a $0.1 \mathrm{~s}$. De acuerdo con resultados de pruebas de vibración ambiental y modelos elementos finitos calibrados del prototipo, el periodo de vibración es representativo del prototipo de dos niveles (Carrillo, 2010a). En la tabla 7 se muestran los resultados, junto con los cocientes porcentuales entre la demanda y la capacidad de resistencia en términos del coeficiente sísmico asociado al estado límite de resistencia $\left(S_{a} / C_{s-m}\right)$.

Tabla 6 Coeficientes sísmicos medidos en los modelos y extrapolados al prototipo, $C_{s}(\mathrm{~g})$

\begin{tabular}{|c|c|c|c|c|c|c|c|}
\hline & \multirow{2}{*}{ Muro } & \multicolumn{3}{|c|}{$C_{s}(g)$ : Modelo } & \multicolumn{3}{|c|}{$C_{s}(g):$ Prototipo } \\
\hline & & $a$ & $m$ & $u$ & $a$ & $m$ & $u$ \\
\hline \multirow{5}{*}{ 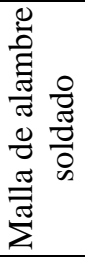 } & MCN50mD & 0.70 & 0.96 & 0.93 & 3.00 & 4.13 & 4.02 \\
\hline & MCC50mD & 0.75 & 1.15 & --- & 3.21 & 4.97 & --- \\
\hline & MVN50mD & 0.73 & 1.00 & 0.79 & 3.14 & 4.30 & 3.39 \\
\hline & \multicolumn{4}{|c|}{ Media aritmética, $\bar{X}$} & 3.12 & 4.47 & 3.70 \\
\hline & \multicolumn{4}{|c|}{ Coeficiente de variación, $C V(\%)$} & 2.9 & 8.0 & 8.5 \\
\hline \multirow{5}{*}{ 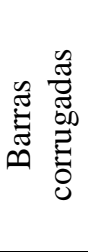 } & MCN100D & 0.67 & 1.12 & 0.87 & 2.86 & 4.83 & 3.75 \\
\hline & MCC100D & 0.76 & 1.20 & 0.96 & 3.28 & 5.16 & 4.13 \\
\hline & MVN100D & 0.74 & 1.23 & 0.94 & 3.19 & 5.29 & 4.05 \\
\hline & \multicolumn{4}{|c|}{ Media aritmética, $\bar{X}$} & 3.11 & 5.09 & 3.98 \\
\hline & \multicolumn{4}{|c|}{ Coeficiente de variación, CV (\%) } & 5.7 & 3.7 & 4.2 \\
\hline \multirow{2}{*}{ 悉 } & \multicolumn{4}{|c|}{ Media aritmética, $\bar{X}$} & 3.11 & 4.78 & 3.87 \\
\hline & \multicolumn{4}{|c|}{ Coeficiente de variación, $C V(\%)$} & 4.5 & 8.9 & 7.0 \\
\hline
\end{tabular}

En la tabla 7 se observa que los valores máximos del cociente $S_{a} / C_{s-m}$ corresponden a suelo firme y son iguales aproximadamente a 39\%, 36\% y 32\% para valores de $Q$ iguales a 1.0, 1.5 y 2.5, respectivamente; es decir, aún para la zona de mayor amenaza sísmica y suponiendo comportamiento elástico $(Q=1.0)$, la demanda sísmica de diseño equivale aproximadamente al $39 \%$ de la capacidad asociada al estado límite de resistencia. Tal como se esperaba, para zonas en las cuales el peligro sísmico en este tipo de viviendas podría ser bajo (suelo blando), el valor del cociente es sustancialmente menor; es decir, aproximadamente $4.0 \%$. Con base en los resultados anteriores, así como los reportados por Flores et al. (2007) y Sánchez (2010), las cuantías mínimas de refuerzo a cortante estipuladas en los reglamentos vigentes para diseño sísmico de viviendas de las características aquí estudiadas son conservadoras, especialmente para estructuras situadas en algunas zonas de la República Mexicana. Por ejemplo, para $a_{0}^{r} \leq 175 \mathrm{~cm} / \mathrm{s}^{2}$, el valor máximo del cociente $S_{a} / C_{s-m}$ es igual aproximadamente a $16 \%$ y $3 \%$ en viviendas construidas sobre suelo firme y blando, respectivamente. Por consiguiente, en dichas zonas se propone prescindir del refuerzo a cortante en el alma del muro, siempre y cuando se establezcan mecanismos adecuados para controlar el agrietamiento por contracción, así como distorsiones permisibles que limiten 
el agrietamiento del concreto para el estado límite de resistencia. En las zonas donde se suprima el refuerzo a cortante del alma de los muros, se propone utilizar los indicadores de desempeño que se indican en la tabla 5. Como se muestra en la tabla, para estos muros se propone que $R_{P(S C)}=0.15 \%$ para el nivel de desempeño de seguridad al colapso y $R_{P(O I)}=0.05 \%$ para ocupación inmediata. Para estos muros, la distorsión permisible del nivel de desempeño de seguridad al colapso está asociada aproximadamente al $25 \%$ de la resistencia al cortante de los muros sin refuerzo en el alma (figura 10) y, como se muestra en la tabla 4, el valor aquí recomendado para estos muros es igual al valor estipulado en NTC-M para muros de mampostería sin confinar y sin reforzar.

Tabla 7 Coeficientes sísmicos para diseño propuestos en la República Mexicana (MDOC-CFE, 2008)

\begin{tabular}{|c|c|c|c|c|c|c|c|c|c|c|c|c|c|c|}
\hline \multirow{2}{*}{ 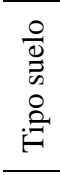 } & \multirow[b]{2}{*}{$\begin{array}{l}T_{S} \\
S\end{array}$} & \multirow{2}{*}{$\rho_{s}$} & \multirow{2}{*}{$R$} & \multirow{2}{*}{$\begin{array}{c}a_{0}^{r} \\
\mathrm{~cm} / \mathrm{s}^{2}\end{array}$} & \multirow{2}{*}{$\begin{array}{c}S_{a e} \\
g\end{array}$} & \multicolumn{3}{|c|}{$Q=1.0$} & \multicolumn{3}{|c|}{$Q=1.5$} & \multicolumn{3}{|c|}{$Q=2.5$} \\
\hline & & & & & & $Q^{\prime}$ & $\begin{array}{c}S_{a} \\
g\end{array}$ & $\begin{array}{c}S_{a} / C_{S-} \\
m \\
\% \\
\end{array}$ & $Q^{\prime}$ & $\begin{array}{c}S_{a} \\
g\end{array}$ & $\begin{array}{c}S_{a} / C_{s-} \\
m \\
\% \\
\end{array}$ & $Q^{\prime}$ & $\begin{array}{c}S_{a} \\
g\end{array}$ & $\begin{array}{c}S_{a} / C_{s-} \\
m \\
\% \\
\end{array}$ \\
\hline \multirow{4}{*}{ 荎 } & \multirow{4}{*}{0.1} & \multirow{4}{*}{0.625} & \multirow{4}{*}{1} & 75 & 0.30 & \multirow{4}{*}{1.00} & 0.30 & 6.3 & \multirow{4}{*}{1.07} & 0.28 & 5.9 & \multirow{4}{*}{1.20} & 0.25 & 5.2 \\
\hline & & & & 175 & 0.74 & & 0.74 & 15.5 & & 0.69 & 14.5 & & 0.62 & 12.9 \\
\hline & & & & 250 & 1.10 & & 1.10 & 23.0 & & 1.03 & 21.5 & & 0.92 & 19.2 \\
\hline & & & & 400 & 1.84 & & 1.84 & 38.5 & & 1.72 & 36.0 & & 1.53 & 32.1 \\
\hline \multirow{4}{*}{$\begin{array}{l}\frac{8}{0} \\
\frac{\pi}{n ! n}\end{array}$} & \multirow{4}{*}{3.0} & \multirow{4}{*}{0.250} & \multirow{4}{*}{1} & 100 & 0.10 & \multirow{4}{*}{1.00} & 0.10 & 2.1 & \multirow{4}{*}{1.02} & 0.10 & 2.1 & \multirow{4}{*}{1.05} & 0.09 & 2.0 \\
\hline & & & & 150 & 0.14 & & 0.14 & 2.9 & & 0.14 & 2.9 & & 0.13 & 2.8 \\
\hline & & & & 175 & 0.15 & & 0.15 & 3.1 & & 0.15 & 3.1 & & 0.14 & 3.0 \\
\hline & & & & 250 & 0.19 & & 0.19 & 4.0 & & 0.19 & 3.9 & & 0.18 & 3.8 \\
\hline
\end{tabular}

$R=$ factor de reducción por sobrerresistencia, $a_{0}^{r}=$ aceleración máxima en roca

$S_{a e}=$ ordenada del espectro elástico de aceleraciones para $T=0.10 \mathrm{~s}$

$S_{a}=$ ordenada del espectro de aceleraciones de diseño para $T=0.10 \mathrm{~s} ; S_{a}=S_{a e} /\left(Q^{\prime} R\right)$

$C_{s}=$ coeficiente sísmico medido (promedio) extrapolado al prototipo para el estado límite de resistencia = 4.78 (tabla 6).

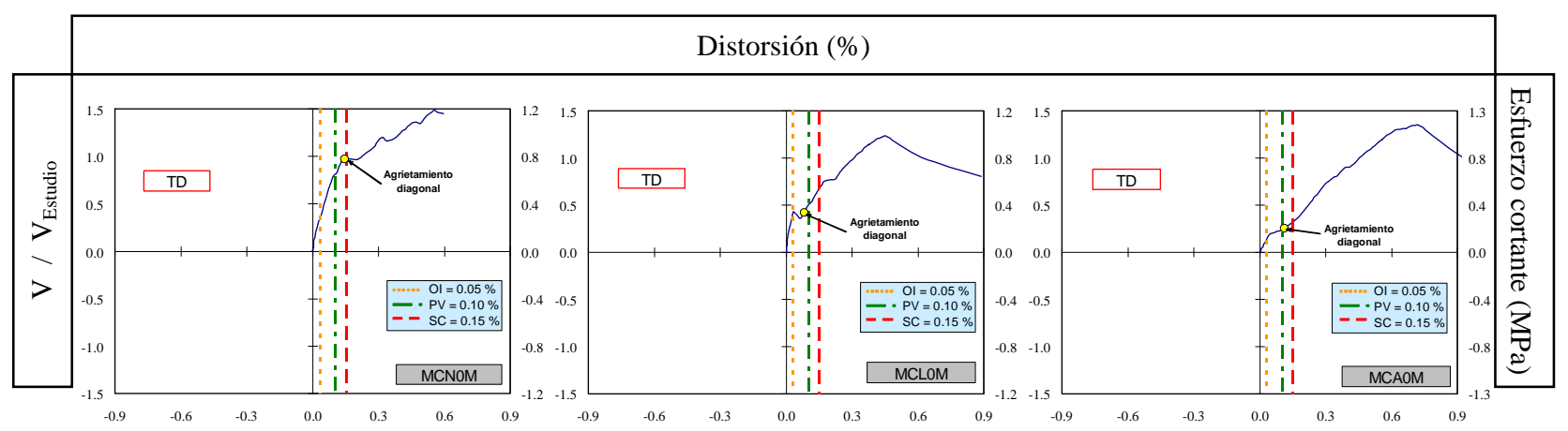

(a) Concreto normal

(b) Concreto ligero

(c) Concreto autocompactable

Figura 10 Distorsiones permisibles en muros sin refuerzo a cortante en el alma $\left(h_{w} / l_{w}=1.0\right)$

\section{Recomendación para cuantías mínimas de refuerzo}

Como se indicó en la sección anterior, desde el punto de vista de resistencia es factible reducir las cuantías de refuerzo a cortante en el alma de los muros en viviendas de las características aquí estudiadas. Adicionalmente, al disminuir las cuantías de refuerzo, el costo de las viviendas se reduce y, de esta manera, mayor número de familias podrán acceder a una vivienda. Con el propósito de brindar a la 
población una vivienda de concreto de mayor calidad, segura ante eventos sísmicos y más económica, en la tabla 8 se indican las recomendaciones para diseño sísmico de las viviendas de concreto de uno y dos niveles. Dichas recomendaciones se establecieron de acuerdo con la capacidad y la demanda de las viviendas; es decir, con un enfoque de diseño por desempeño. La propuesta indicada en la tabla 8 se puede aplicar en un reglamento de diseño, siempre y cuando la resistencia mínima del concreto sea igual a $20 \mathrm{MPa}$ y se cumplan los requisitos de durabilidad preescritos en las normas NMX-C-403 (ONNCCE, 1999) o NMX-C-155 (ONNCCE, 2004b). Esta exigencia se establece con el propósito de mejorar el desempeño del concreto en la vivienda de acuerdo con las metodologías y técnicas de producción disponibles.

Tabla 8 Recomendaciones para diseño de viviendas de baja altura

\begin{tabular}{|c|c|c|c|c|c|c|c|c|c|}
\hline \multirow{2}{*}{$\begin{array}{c}a_{0}^{r} \\
\mathrm{~cm} / \mathrm{s}^{2}\end{array}$} & \multicolumn{5}{|c|}{ Diseño a fuerza cortante } & \multicolumn{2}{|c|}{$\begin{array}{c}\text { Cambios } \\
\text { volumétricos }\end{array}$} & \multirow{2}{*}{$\begin{array}{l}\text { Diseño a } \\
\text { flexión }\end{array}$} & \multirow{2}{*}{$\begin{array}{c}\text { Refuerzo por } \\
\text { integridad } \\
\text { estructural }\end{array}$} \\
\hline & $V_{u} / V_{c}$ & $\begin{array}{c}\rho_{\min } \\
\%\end{array}$ & $\begin{array}{l}\text { Tipo de } \\
\text { refuerzo }\end{array}$ & $Q^{(1)}$ & $\begin{array}{c}R_{\text {perm }} \\
\%\end{array}$ & $\begin{array}{c}\text { Tipo }^{(2)} \\
\text { junta }\end{array}$ & $\begin{array}{c}\rho_{s 1} \\
\%\end{array}$ & & \\
\hline \multirow{2}{*}{$<175$} & $<0.25$ & 0 & --- & 1.0 & 0.15 & 2 & $\begin{array}{ll}-- \\
--\end{array}$ & \multirow{4}{*}{$\begin{array}{l}\text { Se calcula de } \\
\text { forma } \\
\text { convencional }\end{array}$} & \multirow{4}{*}{$\begin{array}{c}\text { Resistir las } \\
\text { componentes } \\
\text { horizontal y } \\
\text { vertical de un } \\
\text { puntal diagonal de } \\
\text { compresión }\end{array}$} \\
\hline & $\geq 0.25$ & 0.12 & Malla $^{(3)}$ & 1.5 & 0.35 & 1 & 0.12 & & \\
\hline $175-250$ & -- & 0.12 & Malla $^{(3)}$ & 1.5 & 0.35 & 1 & 0.12 & & \\
\hline$>250$ & --- & 0.25 & Barras & 2.5 & 0.65 & 1 & 0.12 & & \\
\hline
\end{tabular}

(1) Para el diseño de toda la vivienda se usará el valor mínimo de $Q$

(2) Junta de contracción o aislamiento (tabla 9)

(3) Se podrían utilizar barras corrugadas, pero no se cumpliría el requisito de separación máxima al utilizar barras No 3 y $\rho_{\text {min }}=0.12 \%$.

Los valores límite de $a_{0}^{r}$ que se indican en la tabla $8\left(175\right.$ y $\left.250 \mathrm{~cm} / \mathrm{s}^{2}\right)$ se establecieron a partir del trazado de las zonas B y C definidas en la versión del año 1993 del MDOC-CFE, sobre el mapa de $a_{0}{ }^{r}$ propuesto en la versión del año 2008 del MDOC-CFE. El ACI-318 (2008) también permite utilizar cuantías de refuerzo en el alma del muro menores que $0.25 \%$, siempre y cuando la fuerza cortante de diseño sea menor que $0.083 \sqrt{f_{c}{ }^{\prime}} A_{w}$ en MPa $\left(0.265 \sqrt{f_{c}{ }^{\prime}} A_{w}\right.$ en $\left.\mathrm{kgf} / \mathrm{cm}^{2}\right)$. En este caso, el ACI-318 admite reducir las cuantías mínimas de refuerzo horizontal y vertical a $0.20 \%$ y $0.12 \%$, respectivamente, para barras menores que la No. 5 con $f_{y} \geq 412 \mathrm{MPa}\left(4,200 \mathrm{kgf} / \mathrm{cm}^{2}\right)$ o alambre soldado con diámetro menor o igual que $15.9 \mathrm{~mm}$. Adicionalmente, en el EC8 (2004) se especifica que si $\phi V_{c} \geq V_{u}$ en un muro "robusto" clasificado con comportamiento dúctil moderado, no es necesario disponer la cuantía mínima de refuerzo a cortante en el alma, siempre y cuando $V_{u}$ se incremente en función de un factor que varía aproximadamente entre 1.5 y 2.0. El valor de la cuantía mínima por cambios volumétricos $\left(\rho_{s 1}\right)$ se obtuvo al reemplazar las características específicas de muros de concreto para viviendas de hasta dos niveles, en la expresión especificada en las NTC-C (2004). El valor propuesto de $\rho_{s 1}(0.12 \%)$ es cercano al $50 \%$ de la cuantía mínima a cortante. Los requisitos de las juntas verticales de contracción o aislamiento se presentan en la tabla 9, los cuales se establecieron de acuerdo con las recomendaciones del informe ACI-224 (2008).

Adicionalmente, con objeto de mejorar la redundancia y capacidad de desplazamiento de viviendas de concreto de hasta dos niveles, en todos los muros se debe disponer refuerzo por integridad estructural tal como se especifica en la tabla 8. Siguiendo los lineamientos indicados en la sección 7.13 del ACI-318 (2008) y en Breen (1980), este refuerzo debe estar alojado en secciones rectangulares de concreto reforzado de cuanto menos $100 \mathrm{~mm}$ de lado y debe calcularse de modo que resista las componentes horizontal y vertical de un puntal diagonal de compresión en el muro de concreto con magnitud asociada a la falla del mismo. Sin embargo, no es necesario que el refuerzo vertical por integridad estructural sea mayor que el requerido por las solicitaciones de diseño por flexión. Opcionalmente, el refuerzo por 
integridad estructural se puede calcular de forma similar a lo estipulado en la sección 7.3 de las NTC-M (2004) para muros de mampostería no reforzada.

Tabla 9 Características de las juntas verticales de contracción o aislamiento

\begin{tabular}{|c|c|c|}
\hline $\begin{array}{l}\text { Tipo de } \\
\text { junta }\end{array}$ & Requisitos particulares & Requisitos generales \\
\hline 1 & $\begin{array}{l}\text { a) la separación máxima debe ser } \\
\text { igual a dos veces la altura libre de } \\
\text { entrepiso } \\
\text { b) todo el refuerzo a cortante del } \\
\text { muro debe continuar sobre la junta }\end{array}$ & $\begin{array}{l}\text { a) la profundidad total mínima de la junta debe ser } \\
\text { igual al } 25 \% \text { del espesor del muro; es decir, } 12.5 \% \\
\text { de profundidad en cada cara para muros con ambas } \\
\text { caras descubiertas } \\
\text { b) la profundidad total máxima de la junta debe ser }\end{array}$ \\
\hline 2 & $\begin{array}{l}\text { a) la separación máxima debe ser } \\
\text { igual a la altura libre de entrepiso } \\
\text { b) se deben colocar juntas en línea } \\
\text { con los bordes de todas las } \\
\text { aberturas }\end{array}$ & $\begin{array}{l}\text { igual al 50\% del espesor del muro } \\
\text { c) se deben utilizar selladores de junta flexibles para } \\
\text { prevenir que penetre humedad o químicos que } \\
\text { puedan promover la corrosión del refuerzo y/o la } \\
\text { degradación del concreto. }\end{array}$ \\
\hline
\end{tabular}

Como se indica en la tabla 8, los requisitos de diseño a fuerza cortante dependen básicamente de la amenaza sísmica en el sitio, del cociente entre la fuerza cortante de diseño y la contribución del concreto a la resistencia $\left(V_{u} / V_{c}\right)$, y del tipo de refuerzo a cortante en el alma en el muro. La capacidad de resistencia a fuerza cortante $\left(V_{c}+V_{s}\right)$, las cuantías de refuerzo horizontal y vertical $\left(\rho_{h} \mathrm{y} \rho_{v}\right) \mathrm{y}$, las distorsiones permisibles $\left(R_{P}\right)$, también se deben estimar de acuerdo con las recomendaciones aquí propuestas. La verificación de estas recomendaciones se llevó a cabo a partir del análisis y diseño de ocho prototipos de vivienda de uno y dos niveles ubicados en diferentes zonas de la República Mexicana (Carrillo, 2010a). A partir de la respuesta medida en los especímenes y los resultados de modelación de prototipos de vivienda, se considera que las recomendaciones aquí propuestas son adecuadas para fines de diseño reglamentario.

\section{DISCUSIÓN DE RESULTADOS}

Las ecuaciones propuestas son de carácter determinista e incluyen las características particulares de muros en viviendas de baja altura en México. En general, las ecuaciones están destinadas a capturar la respuesta promedio de la base de datos utilizada y se desarrollaron a partir de un análisis de regresión no lineal iterativo. Los valores aquí recomendados de $R_{P}$ están asociados a niveles de seguridad adecuados para su aplicación en la práctica de la ingeniería estructural y, por tanto, no representan la capacidad máxima de los especímenes estudiados.

La aplicación de las ecuaciones está limitada a muros de concreto para vivienda de uno y dos niveles; es decir, el modelo desarrollado simula apropiadamente el comportamiento sísmico de muros con relación de aspecto menor o igual que 2.0 y muros con aberturas; muros cuya respuesta está gobernada por las deformaciones de corte; muros construidos con concreto de peso normal, peso ligero y autocompactable; resistencia a la comprensión del concreto entre 15 y $25 \mathrm{MPa}$; muros con esfuerzo axial menor que $3 \%$ de $f_{c}$ '; cuantías de refuerzo en el alma menores o iguales que $0.25 \%$; refuerzo en el alma del muro conformado por barras corrugadas o malla de alambre soldado y con la misma cuantía de refuerzo horizontal y vertical en el alma. 


\section{CONCLUSIONES}

Con base en los resultados de un extenso programa de investigación analítico y experimental, se desarrollaron ecuaciones para estimar la capacidad de resistencia al cortante de muros de concreto con las características particulares de viviendas de baja altura. Adicionalmente, se presentaron recomendaciones para estimar los parámetros principales para diseño sísmico reglamentario (factor de comportamiento sísmico y distorsiones permisibles de entrepiso). Las ecuaciones y los parámetros se plantearon de tal manera que puedan adaptarse fácilmente al formato de un reglamento de diseño, por el ejemplo, al Reglamento del Distrito Federal. Sin embargo, si se considera que el énfasis del diseño sísmico está cambiando a un enfoque por desempeño, los indicadores de desempeño propuestos también son una herramienta robusta para que los diseñadores y desarrolladores de códigos evalúen la efectividad de los procedimientos de diseño disponibles.

A partir del análisis de los cocientes entre la demanda y la capacidad de resistencia de las viviendas estudiadas, se observó que aún para la zona de mayor amenaza sísmica y suponiendo comportamiento elástico, la demanda sísmica de diseño fue equivalente al 39\% de la capacidad asociada al estado límite de resistencia. Tal como se esperaba, para zonas en las cuales el peligro sísmico en este tipo de viviendas podría ser bajo (suelo blando), el valor del cociente fue significativamente menor, es decir, aproximadamente $4.0 \%$. Por tanto, se comprobó que las cuantías mínimas de refuerzo a cortante estipuladas en los reglamentos disponibles para diseño sísmico de viviendas de las características aquí estudiadas, son conservadoras o muy conservadoras, especialmente para estructuras situadas en algunas zonas de la República Mexicana. Con base en lo anterior, se recomendaron valores de las cuantías mínimas de refuerzo a cortante, de acuerdo con la capacidad y la demanda de las viviendas; es decir, con un enfoque de diseño por desempeño. Al adoptar las recomendaciones aquí propuestas, se espera que el diseño sísmico promueva la construcción de una vivienda de concreto de mayor calidad, segura ante eventos sísmicos y más económica.

\section{AGRADECIMIENTOS}

Los autores agradecen al Grupo CEMEX por el apoyo económico de la investigación, en especial, a los Ingenieros Roberto Uribe y Ángel Ponce, Director General y Gerente de la División de Estructuras, respectivamente, del Centro de Tecnología del Cemento y del Concreto, CEMEX, así como al personal de los laboratorios del Instituto de Ingeniería de la UNAM, por su valiosa colaboración durante la realización de los ensayos. El contenido de este artículo representa exclusivamente la opinión de los autores y no refleja la opinión de los patrocinadores y/o colaboradores.

\section{REFERENCIAS}

ACI - Comité 318 (2008), "Building Code Requirements for Structural Concrete (ACI 318-08) and Commentary (ACI 318R-08)”, American Concrete Institute, Farmington Hills, MI, EUA, 465 pp.

ACI - Comité 224 (2008), “Joints in concrete construction (ACI 224.3R-95)”, American Concrete Institute, Farmington Hills, MI, EUA, 42 pp.

Breen, J (1980), “Developing structural integrity in bearing wall buildings”, PCI Journal, Vol. 25, No. 1, pp. 42-73. 
Carrillo, J (2010a), "Evaluación del comportamiento al cortante de muros de concreto para vivienda por medio de ensayos dinámicos”, Tesis de Doctorado, Universidad Nacional Autónoma de México, 475 pp.

Carrillo, J (2010b), "Diseño sísmico de VIS con muros de concreto”, Revista de la Escuela Colombiana de Ingeniería, Vol. 80, Octubre - Diciembre, pp. 7-17.

Carrillo, J y S Alcocer (2011a), "Propiedades dinámicas de viviendas construidas con muros de concreto”, Serie Investigación y Desarrollo, SID/671, Instituto de Ingeniería, UNAM, 81 pp.

Carrillo, J y S Alcocer (2011b), "Improved external device for a mass-carrying sliding system for shaking table testing”, Journal of Earthquake Engineering and Structural Dynamics, Vol. 40, No. 4, pp. 393-411.

Carrillo, J y S Alcocer (2010), "Efectos del protocolo de ensayo sobre la respuesta de muros de concreto con relación de aspecto igual a uno”, XVII Congreso Nacional de Ingeniería Estructural, León, México, Tema VI, Artículo 1.

Carrillo, J, S Alcocer y R Uribe (2009), “Comportamiento dinámico y cuasi-estático de sistemas estructurales de muros de concreto con aberturas”, XVII Congreso Nacional de Ingeniería Sísmica, Puebla, México, Tema VIII, Artículo 1.

Duffey T, C Farrar y A Goldman (1994a). "Low-rise shear wall ultimate drift limits”. Journal of Earthquake Spectra, Vol. 10, No. 4, pp. 655-674.

Duffey T, A Goldman y C Farrar (1994b). "Shear wall ultimate drift limits". Informe No. NUREG/CR6104, Los Alamos National Laboratory. Preparado para: U.S. Nuclear Regulatory Commission. Washington, DC, EUA.

Eberhard M, S Baldridge, J Marshall, W Mooney y G Rix (2010), "The Mw 7.0 Haiti earthquake of January 12, 2010”, V1.1: USGS/EERI Advance Reconnaissance Team”, U.S. Geological Survey and Earthquake Engineering Research Institute. California, EUA, pp. 56.

Flores, L, S Alcocer, J Carrillo, A Sánchez, R Uribe y A Ponce (2007), "Ensayo de muros de concreto con diferente relación de aspecto y bajas cuantías de refuerzo, para uso en vivienda”, $X V I$ Congreso Nacional de Ingeniería Sísmica, Ixtapa-Zihuatanejo, Guerrero, México, Tema XI, Artículo 2.

Ghobarah A (2004), “On drift limits associated with different damage levels”, International Workshop on Performance-Based Seismic Design Concepts and Implementation, Bled, Slovenia.

Johansson J, P Mayorca, E Leon y A Torres (2007), "Pisco earthquake, Peru, August 15, 2007: JSCE/JAEE/UT Investigation Team”, Japan Association of Earthquake Engineering. Japón, pp. 109.

Leiva G y E Montaño (2001), "Resistencia al corte de muros de hormigón armado”, Revista de Ingeniería Sísmica, No. 64, México, pp. 1-18.

MDOC-CFE (2008), "Manual de diseño de obras civiles: Diseño por sismo", Comisión Federal de Electricidad - CFE, México, 324 pp.

MDOC-CFE (1993), "Manual de diseño de obras civiles: Diseño por sismo”, Comisión Federal de Electricidad - CFE, México.

NMX-B-253 (2006), "Norma Mexicana: Alambre de acero liso o corrugado para refuerzo de concreto”, ONNCCE-CANACERO, México, 8 pp.

NMX-C-155 (2004), “Norma Mexicana: Especificaciones para concreto hidráulico industrializado”, ONNCCE, México, 24 pp. 
NMX-C-403 (1999), “Norma Mexicana: Concreto hidráulico para uso estructural”, ONNCCE, México, 32 pp.

NTC-C (2004), "Normas técnicas complementarias para diseño y construcción de estructuras de concreto”, Gaceta Oficial del Distrito Federal, México, 101 pp.

NTC-M (2004), “Normas técnicas complementarias para diseño y construcción de estructuras de mampostería”, Gaceta Oficial del Distrito Federal, México, 48 pp.

NTC-S (2004), “Normas técnicas complementarias para diseño por sismo”, Gaceta Oficial del Distrito Federal, México, 22 pp.

Priestley M (2000), "Performance based seismic design”, 12th World Conference on Earthquake Engineering, Nueva Zelanda, Paper 2831.

Pujol S, J Ramírez y A Sarria A (1999), “Coffee zone Colombia, January 25 Earthquake: Observations on the behavior of low-rise reinforced concrete buildings", http://nisee.berkeley.edu/lessons/colombia.pdf, pp. 9.

Rico, A, J Carrillo y S Alcocer (2011), "Propiedades mecánicas de la malla de alambre soldado disponible en la Zona Metropolitana de la Ciudad de México”, Serie Investigación y Desarrollo, SID/669, Instituto de Ingeniería, UNAM, 55 pp.

Rosenblueth E, R Gómez y J Ávila J. (1991), “Comentarios y ejemplos a las normas técnicas complementarias para diseño por sismo, DF”, Reporte Técnico No. ES7, Instituto de Ingeniería, Universidad Nacional Autónoma de México, UNAM. México.

Sánchez A (2010), “Comportamiento sísmico de viviendas construidas con muros de concreto”, Informe Técnico, Instituto de Ingeniería, Universidad Nacional Autónoma de México, UNAM. México.

Tomazevic M y T Velechovsky (1992), "Some aspects of testing small-scale masonry building model on simple earthquake simulator”, Journal of Earthquake Engineering and Structural Dynamics, Vol. 21, No. 11, pp. 945-963.

Wood S (1990), "Shear strength of low-rise reinforced concrete walls". ACI Structural Journal, Vol. 87, No. 1, pp. 99-107. 